OPEN ACCESS

Edited by:

Pinarosa Avato,

University of Bari Aldo Moro, Italy

Reviewed by:

Johanna Mahwahwatse Bapela, University of Pretoria, South Africa

Leonardo Borges,

State University of Goiás, Brazi

*Correspondence:

Antoniana U. Krettli

antoniana.krettli@fiocruz.br

Specialty section: This article was submitted to

Ethnopharmacology,

a section of the journal

Frontiers in Pharmacology

Received: 30 June 2021

Accepted: 31 August 2021

Published: 22 September 2021

Citation:

Ceravolo IP, Aguiar AC, Adebayo JO and Krettli AU (2021) Studies on

Activities and Chemical

Characterization of Medicinal Plants in Search for New Antimalarials: A Ten Year Review on Ethnopharmacology.

Front. Pharmacol. 12:734263.

doi: 10.3389/fphar.2021.734263

\section{Studies on Activities and Chemical Characterization of Medicinal Plants in Search for New Antimalarials: A Ten Year Review on Ethnopharmacology}

\author{
Isabela P. Ceravolo ${ }^{1}$, Anna C. Aguiar ${ }^{2}$, Joseph O. Adebayo ${ }^{3}$ and Antoniana U. Krettli ${ }^{1 *}$ \\ ${ }^{1}$ Instituto René Rachou, Fundação Oswaldo Cruz (Fiocruz), Belo Horizonte, Brazil, ${ }^{2}$ Departamento de Biociência, Universidade \\ Federal de São Paulo, Santos, Brazil, ${ }^{3}$ Department of Biochemistry, University of Ilorin, Ilorin, Nigeria
}

Malaria is an endemic disease that affected 229 million people and caused 409 thousand deaths, in 2019. Disease control is based on early diagnosis and specific treatment with antimalarial drugs since no effective vaccines are commercially available to prevent the disease. Drug chemotherapy has a strong historical link to the use of traditional plant infusions and other natural products in various cultures. The research based on such knowledge has yielded two drugs in medicine: the alkaloid quinine from Cinchona species, native in the Amazon highland rain forest in South America, and artemisinin from Artemisia annua, a species from the millenary Chinese medicine. The artemisinin-based combination therapies (ACTs), proven to be highly effective against malaria parasites, and considered as "the last bullet to fight drug-resistant malaria parasites," have limited use now due to the emergence of multidrug resistance. In addition, the limited number of therapeutic options makes urgent the development of new antimalarial drugs. This review focuses on the antimalarial activities of 90 plant species obtained from a search using Pubmed database with keywords "antimalarials," "plants" and "natural products." We selected only papers published in the last 10 years (2011-2020), with a further analysis of those which were tested experimentally in malaria infected mice. Most plant species studied were from the African continent, followed by Asia and South America; their antimalarial activities were evaluated against asexual blood parasites, and only one species was evaluated for transmission blocking activity. Only a few compounds isolated from these plants were active and had their mechanisms of action delineated, thereby limiting the contribution of these medicinal plants as sources of novel antimalarial pharmacophores, which are highly necessary for the development of effective drugs. Nevertheless, the search for bioactive compounds remains as a promising strategy for the development of new antimalarials and the validation of traditional treatments against malaria. One species native in South America, Ampelozyzyphus amazonicus, and is largely used against human malaria in Brazil has a prophylactic effect, interfering with the viability of sporozoites in in vitro and in vivo experiments.

Keywords: malaria, antimalarials, medicinal plants, natural products, in vivo tests, ethnopharmacology 


\section{INTRODUCTION}

Human malaria is an infectious disease caused by single-cell protozoan parasites of the Plasmodium genus, namely: $P$. falciparum, $P$. vivax, $P$. ovale, $P$. malariae, and $P$. knowlesi, and is transmitted through the bite of female Anopheles mosquitoes (Crompton et al., 2014; Singh et al., 2021); of these, $P$. falciparum is the most virulent and prevalent globally. Symptoms can range from a mild to severe disease which can cause important physical disabilities and death, with an enormous health burden, especially among the most vulnerable and poor populations. While $P$. falciparum is responsible for most deaths, $P$. vivax is the most widespread of all of the Plasmodium species, can cause severe, even fatal infections and results in significant global morbidity and mortality (Menkin-Smith and Winders, 2020). Recent studies have reported the virulence of $P$. vivax (Lampah et al., 2011; Tanwar et al., 2011); although, the proportion of cases due to $P$. vivax reduced from about
$7 \%$ of the global malaria cases in 2000 to $3 \%$ in 2019 (WHO, 2020a).

Malaria is an endemic disease in the tropical and subtropical regions of the world. An estimated 229 million new cases and 409,000 deaths were recorded in 2019 (WHO, 2020a). About $94 \%$ of the global malaria cases and $95 \%$ of the deaths were recorded in the African region in 2019 (Figure 1), with children under the age of 5 years and pregnant women being the most susceptible groups (WHO, 2020a). Nigeria was responsible for $27 \%$ of the malaria cases and $23 \%$ of deaths globally in 2019 (WHO, 2020a); this high prevalence illustrated in Figure 2 (WHO, 2018a). This is because Nigeria is the most populous nation in Africa, with $P$. falciparum being the main cause of malaria (Adebayo and Krettli, 2011; WHO, 2020a). Actually, P. falciparum is the most predominant species in every country in sub-Saharan Africa; the countries with the highest number of malaria cases in West, East, Central and Southern Africa are shown in Table 1. In some countries in Africa, a hundred percent of the population is at risk of the disease (both low and high), such as Nigeria, Mozambique,

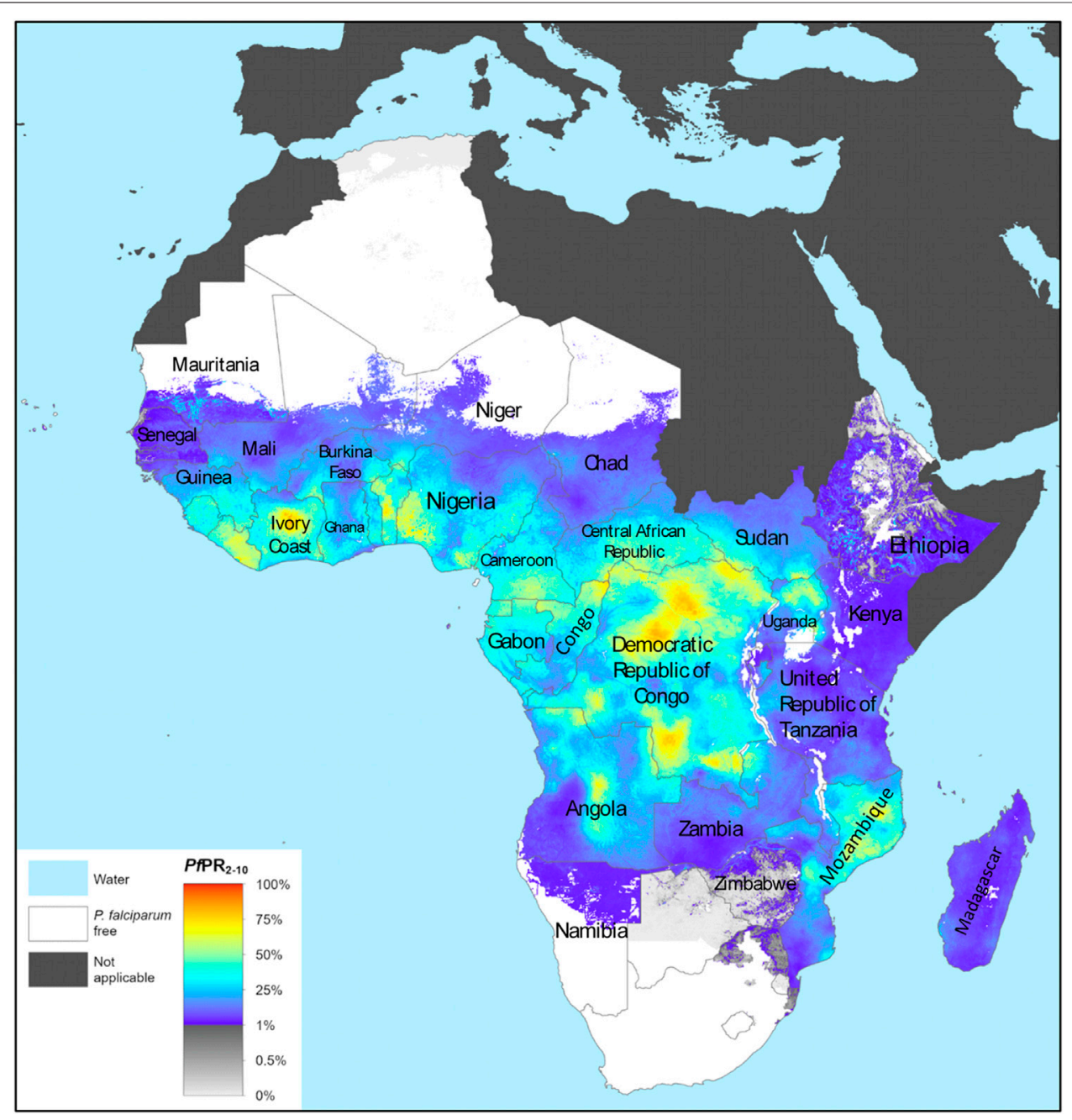

FIGURE 1 | The spatial distribution of Plasmodium falciparum malaria endemicity in 2019 in the WHO African Region. Adapted from The Malaria Atlas Project (WHO, 2020b). Source: https://malariaatlas.org/trends/region. 

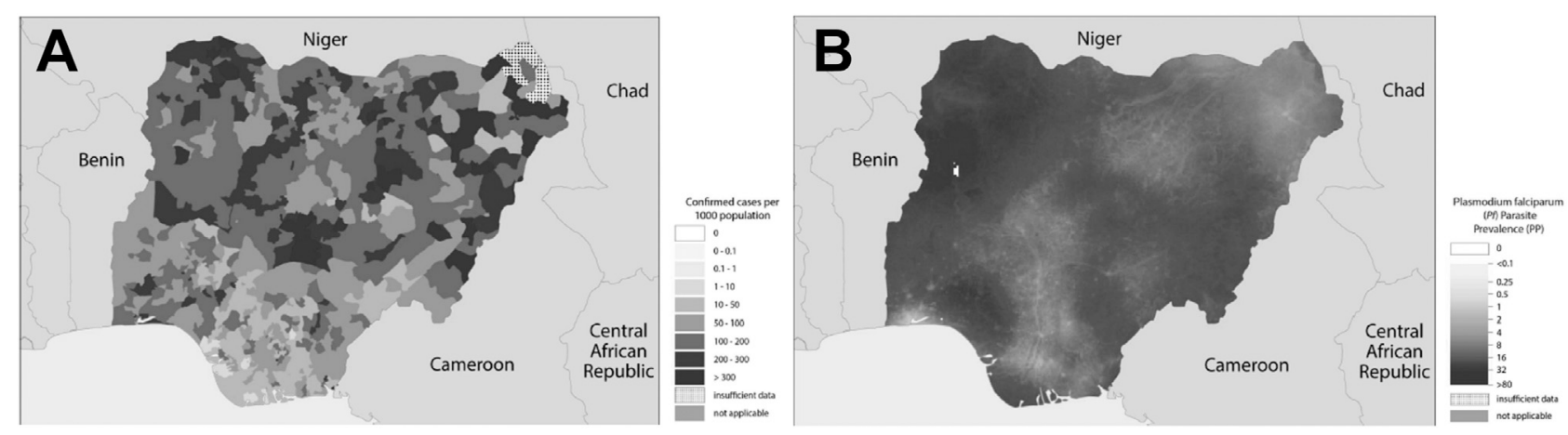

FIGURE 2 | (A) Confirmed cases of malaria in Nigeria in 2017; (B) Plasmodium falciparum prevalence in Nigeria in 2017. Source: Adapted from WHO (2018a).

TABLE 1 | Population, estimated malaria cases and deaths in selected countries with the highest malaria cases in different African regions in 2019.

\begin{tabular}{|c|c|c|c|c|c|c|}
\hline $\begin{array}{l}\text { Region of } \\
\text { Africa }\end{array}$ & Country & $\begin{array}{c}\text { Total } \\
\text { population }\end{array}$ & $\begin{array}{c}\text { Population at } \\
\text { risk (\% } \\
\text { of total } \\
\text { population) }\end{array}$ & $\begin{array}{c}\text { Cases (\% } \\
\text { of population } \\
\text { at risk) }\end{array}$ & Deaths & $\begin{array}{l}\text { Most predominant } \\
\text { Plasmodium species }\end{array}$ \\
\hline East Africa & Mozambique & $30,366,043$ & $30,366,043(100)$ & $\begin{array}{c}9,364,806 \\
(30.8)\end{array}$ & 14,971 & P. falciparum \\
\hline Central Africa & $\begin{array}{l}\text { Democratic Republic of } \\
\text { Congo }\end{array}$ & $86,790,564$ & $86,790,564(100)$ & $\begin{array}{c}28,280,007 \\
(32.6)\end{array}$ & 44 & P. falciparum \\
\hline $\begin{array}{l}\text { Southern } \\
\text { Africa }\end{array}$ & Namibia & $2,494,524$ & 1,980,028 (79.4) & $5,618(0.3)$ & 14 & P. falciparum \\
\hline
\end{tabular}

Source: Adapted from WHO (2020a).

Democratic republic of Congo etc. (WHO, 2020a; Table 1). However, the numbers of reported cases in such countries are low compared to the expected numbers because of lack of access to diagnosis/health facilities or presumptuous treatment (Table 1). Ethiopia has been reported to have the highest number of $P$. vivax infections in Africa (WHO, 2020a). The higher prevalence of the disease based on the reported cases in the other regions of Africa compared to Southern Africa (Table 1) is an indication of favorable environmental conditions such as higher temperature and rainfall in such regions (Greenwood et al., 2008).

Drug chemotherapy has a strong historical link to the use of traditional plant infusions in various cultures. Research based on such knowledge has yielded the two most important drugs critical to malaria: the alkaloid quinine present in Cinchona species of highland rain forests in South America and artemisinin from Artemisia annua, first registered in the millenary Chinese medicine.

Artemisinin-based combination therapies (ACTs) are the most recommended treatments for uncomplicated $P$. falciparum malaria, while artesunate is considered the most effective antimalarial drug for severe cases (Roussel et al., 2017), with several biochemical processes reported as targets in the parasites (Wicht et al., 2020). Despite the safety and efficacies proven for the use of these antimalarial drugs, the emergence of multidrug resistance has unfortunately limited their effects and challenged the field (Nieves et al., 2016). The resistance to ACTs is already spreading from Southeast Asia as reported in 2008 (Noedl et al., 2008), giving rise to a danger alert to other high-poverty regions in the world. The identified artemisinin resistance phenotype is associated with mutation of a kelch domain protein gene (k13), postulated to be involved in the parasite protein trafficking organelles during the intraerythrocytic cycle (Ariey et al., 2014). This mutation has recently been reported in East Africa (Uwimana et al., 2020; Asua et al., 2021), Papua New Guinea (Miotto et al., 2020), and French Guiana (Mathieu et al., 2020); yet, there is limited evidence of delayed parasite clearance after ACT treatment in these regions (Yeka et al., 2016).

The treatment failure associated with ACTs in Africa has been linked to resistance of parasite to the partner drugs and not to artemisinin resistance (Slater et al., 2016). However, Ala675Val mutation in the kelch 13 propeller gene was seen in $5 \%$ of isolates in Northern Uganda and this mutation has been known to cause delayed parasite clearance in Southeast Asia (Asua et al., 2019). In the African continent, four countries have identified mutations associated with therapeutic failure of artemisinin derivatives. However, Pfk13 mutations (M476I, P553L, R561H, P574L, 
C580Y, and A675V) showed low frequencies and with no reports of clinical treatment failure, except in Rwanda (Miotto et al., 2020; Uwimana et al., 2020; Asua et al., 2021).

Due to the resistance and the high cost of the potent ACTs, the poor populations of the African continent rely heavily on herbal remedies based on historical and cultural beliefs (Adebayo and Krettli, 2011). In addition, the limited number of therapeutic options makes the development of new antimalarial drugs urgent. The search for bioactive compounds from plants remains a promising strategy for the development of new antimalarial candidates as well as the validation of traditional treatments. In such context, the aim of this study was to review the literature for activities and safety of antimalarial plants in vivo and their isolated active principles studied during the last 10 years.

\section{History of Natural Products and Their Applications in the Treatment of Human Malaria}

The history of medicine dates back to the existence of human civilization. Most new drugs have been generated from natural products (secondary metabolites) and from compounds derived from natural products (Patwardhan et al., 2004; Lahlou, 2007). Natural products from plants, animals, and minerals, have been the basis of treatment of human diseases (Lahlou, 2007); nevertheless, plants used in traditional medicine may hold the key of many potent antimalarial drugs (Rasoanaivo et al., 2004).

Studies of plants used in traditional medicine for the treatment of malaria in various cultures have yielded important findings that are crucial to modern medicine. Two of the most effective drugs for malaria originated from traditional medicine: quinine from bark of Peruvian Cinchona tree, and artemisinin from the Chinese antipyretic Artemisia annua (Rasoanaivo et al., 2004).

Currently, standardized antimalarial phytomedicines are officially commercialized in various malaria endemic countries over the world, namely China, Ghana, India, Mali, and Burkina Faso (Abay et al., 2015), supporting their use as complementary tools to the conventional antimalarial interventions or as alternative treatments in the absence of antimalarial drugs (Willcox, 2011). Examples of herbal therapies are represented by Qing hao (Artemisia annua, Democratic Republic of Congo trials), Totaquina (Cinchona spp., Multicounty trials) and Phyto-laria (Cryptolepis sanguinolenta, Ghana trial) (Willcox, 2011). Medicinal plants are also used to treat malaria in several African countries (Soh and Benoit-Vical, 2007; Pillay et al., 2008; Adebayo and Krettli, 2011; Maranz, 2012; Chinsembu, 2015; Memvanga et al., 2015; Omara et al., 2020). In Ethiopia, $80 \%$ of the rural populations rely on medicinal plants to treat diseases due to the high cost of drugs, low access to health services, among other factors. As a result, many species of medicinal plants are used against malaria there, such as Artemisia annua, Ajuga remota, Azadirachta indica, Argemone mexicana, Vernonia amygdalina, Asparagus africanus, Uvaria leptocladon, and Gossypium spp (Alebie et al., 2017).

\section{Quinine}

Historical records show that in 1638, the countess of Chinchón, wife of the Spanish viceroy in Peru, suffered a severe fever, which

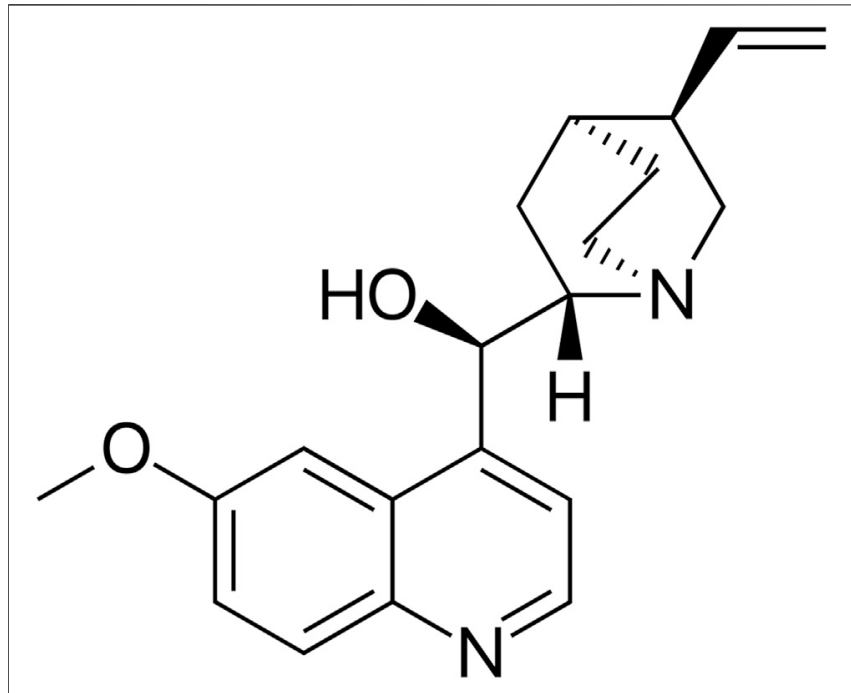

FIGURE 3 | Chemical structure of quinine. Source: https://commons. wikimedia.org/wiki/File:Quinine_structure.svg.

was later known as malaria. When ingesting a portion made by the Indians called "quina-quina" the fever subsided, and the continuity of treatment left her cured (Willcox et al., 2004; Ruiz-Irastorza and Khamashta, 2008; de Oliveira and Szczerbowski, 2009). There is also a reference that the use of Cinchona species in medical practice came to Belgium in 1643, when a public health official in Ghent recommended a powder for the treatment of tertian fevers (Jarcho and Torti, 1993). However, the first indisputable reference is the Schedula Romana, a hand bill issued by the Pharmacy of the Collegio Romano in 1649 and again in 1651, containing precise instructions on its dosage and administration (Jaramillo-Arango, 1949).

Before 1820, the bark of a tree native to South America named Cinchona, was dried, ground to a fine powder, and mixed into a liquid (commonly wine) before being used (Achan et al., 2011). The Jesuit priests of the Spanish mission took the tree bark to Europe to sell its powder as medicine against intermittent fevers. Later on, two French chemists, Pelletier and Caventou (1820), isolated the active principle by precipitation and crystallization and discovered that the base febrifuge compounds were the alkaloids named cinchonine and quinine. From it, the alkaloids quinidine and cinchonidine were also described (Willcox et al., 2004; Kaufman and Ruveda, 2005). The four alkaloids from Cinchona bark were all effective against malaria, but quinidine and quinine possessed equal febrifugal activity, while cinchonidine was slightly less efficacious. An additional 25 alkaloids related to quinine had been isolated by 1884 and six more were isolated between 1884 and 1941 (Turner and Woodward, 1998). By the turn of the 18th and 19th centuries, Cinchona bark and quinine became widely accepted and used instead of the powdered bark as the reference treatment for intermittent fevers throughout the world (Gachelin et al., 2017).

During the second World War, there was an interruption in the supply of Cinchona bark, which contained a high amount of quinine, from the Java island where the seeds were largely 
cultivated (Howard, 1931). In the mid-1800s, the structure of quinine (Figure 3) was elucidated (Kaufman and Ruveda, 2005). Quinine remained the mainstay of malaria treatment until the 1920s, when more effective synthetic antimalarial drugs became available (AdenAbdi et al., 1995). However, the extraction of quinine from the bark of Cinchona does not yield as much as the extraction from the entire tree. The commercial achievement of this bark, almost led to the extinction of the Amazonian trees, and therefore, there was a great need to synthesize the active compounds (Smith and Williams, 2008).

Quinine is an alkaloid that presents rapid schizonticidal action against intra-erythrocytic malaria parasites; it is also gametocytocidal for Plasmodium vivax and Plasmodium malariae, though there are conflicting reports on its gametocytocidal effect on Plasmodium falciparum (Chutmongkonkul et al., 1992; Tanaka and Williamson, 2011). Quinine has also an analgesic effect, but not antipyretic properties, and prevents the hemozoin crystals from growing by intercalating the quinolone rings between the aromatic groups of the ferriprotoporphyrine molecules (Pelletier and Caventou, 1820; Institute of Medicine, 2004; Weissbuch and Leiserowitz, 2008; Gachelin et al., 2017). For centuries, quinine has been used to treat malaria, and it remains the drug of choice in the treatment of severe malaria; it has been chosen as a second line treatment in combination with antibiotics, after artemisinin combination therapy (ACT) in the treatment of complicated malaria (WHO, 2015).

\section{Resistance to Quinine}

Parasite drug resistance is probably the greatest problem faced by malaria control programs worldwide and is an important public health concern. Over the years, malaria parasites have developed resistance to several commonly used antimalarial drugs (Achan et al., 2011). Diminished sensitivity of $P$. falciparum to quinine has been widely documented in Asia (Sucharit et al., 1979; Bjorkman and Phillips-Howard, 1990; Mayxay et al., 2007), South America (Neiva, 1987; Best Plummer and Pinto Pereira, 2008; Legrand et al., 2008), and Africa (Pradines et al., 1998; Zalis et al., 1998; Mutanda, 1999). However, the development of resistance to quinine has been slow; although its use started in the 17th century, resistance to quinine was first reported in 1910 (Peters, 1982), and after so many years of its discovery, quinine remains an important and effective treatment for malaria today, despite sporadic reports of resistance (Institute of Medicine, 2004). Thus, with increasing resistance in almost all areas with $P$. falciparum malaria to synthetic antimalarials by the 1980 s, quinine again played a key role, particularly in the treatment of severe malaria (AdenAbdi et al., 1995).

Quinine is still used as monotherapy to treat malaria today in most countries of Africa and in some Central American, Caribbean, Eastern Mediterranean, South-East Asian and Western Pacific countries (WHO, 2019). In several settings, the use of quinine for uncomplicated malaria cases has reduced due to toxicity, poor compliance, and the implementation of newer and better tolerated therapies. However, from frequent stock-outs of the recommended ACT and the increasing resistance to chloroquine and antifolates,

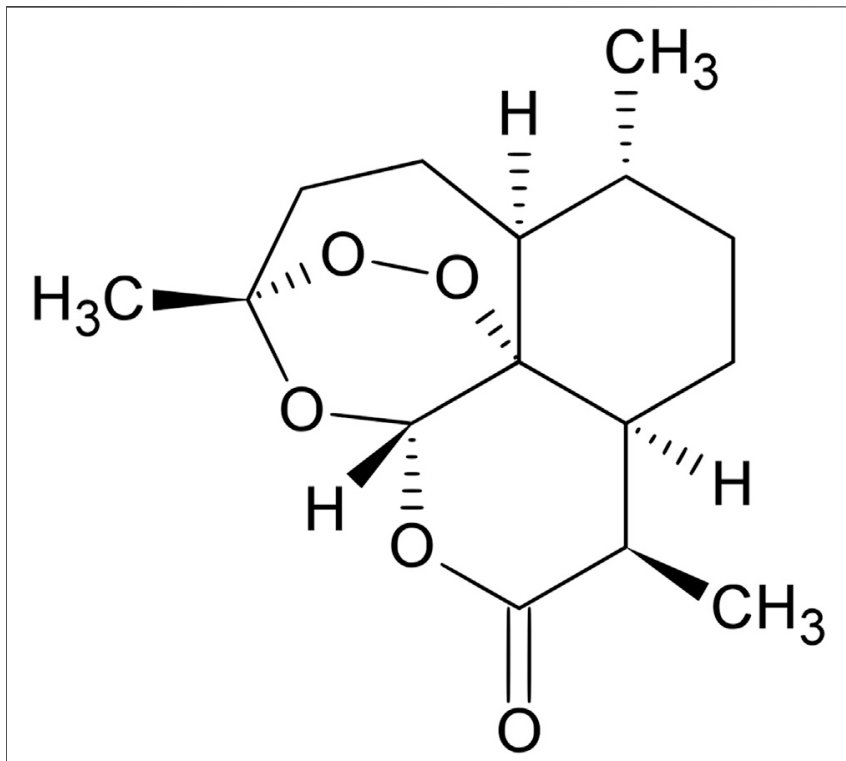

FIGURE 4 | Chemical structure of artemisinin. Source: https://en. wikipedia.org/wiki/Artemisinin.

quinine use in recent times has increased (Wasunna et al., 2008; UMSP, 2010).

The 2015 World Health Organization (WHO) guidelines recommend a combination of quinine and clindamycin, or only quinine to treat pregnant women with uncomplicated $P$. falciparum and who have chloroquine-resistant $P$. vivax malaria during the first trimester. For the treatment of severe malaria of adults and children, it is essential that full doses of effective parenteral (or rectal) antimalarial treatment be given promptly in the initial treatment (WHO, 2015). The Cinchona alkaloids quinine and quinidine are the second choice, after artemisinin derivatives. When a complete oral treatment of severe malaria is not possible, but injections are available, the intramuscular quinine could be used if artesunate or artemether is not available (WHO, 2015).

\section{Artemisinin}

Qinghao ("blue-green herb") is the Chinese name for a relatively common herbal plant otherwise known as Artemisia annua or sweet wormwood (Maude et al., 2010). Artemisia annua is native to Asia (China, Japan, Korea, Vietnam, Myanmar, Northern India, Southern Siberia and throughout eastern Europe) (WHO, 1998), but it also grows in other countries such as Congo, India, Brazil, Australia, Argentina, Bulgaria, France, Hungary, Italy, Spain, and the USA (Willcox et al., 2003; Zhou et al., 2014). There is an integral interaction between plants, the environment and the production of secondary metabolites, which can directly interfere with their quantities and qualities (Kutchan, 2001). The artemisinin content is affected by several factors, such as geographic conditions, harvest time, temperature and fertilizer application; harvesting the plant at the right time is extremely important to ensure the optimal artemisinin content in A. annua. Thus, for the cultivation of this medicinal plant, it is important to 
plan the crop establishment for the beginning of the rainy season, and the soil needs to be plowed to a fine slope and consolidated by rolling when appropriate, because the sowing depth is also critical for A. annua (Jelodar et al., 2014). This means that not all $A$. annua plants have high concentrations of antimalarial compounds.

The A. annua has been used as a remedy by Chinese herbalists for the crafting of aromatic wreaths, as a source of essential oils used in flavoring vermouth, and for more than 2,000 years in Chinese traditional medicine, as a treatment for fever and hemorrhoids (Van Agtmael et al., 1999; Klayman, 1985). Artemisia annua is also used as a source of artemisinin (Zhou et al., 2014), a potent antimalarial drug discovered by the Chinese scientists led by Youyou Tu, that was awarded the 2011 Lasker Prize and the Nobel Prize in Physiology or Medicine in 2015 (Youyou, 2015). The project leading to the discovery of artemisinin was initiated in response to a request from North Vietnamese leaders who were suffering heavy losses of soldiers due to malaria during the Vietnam War (Cui and Su, 2009). The drug is present in the leaves and flowers of the plant accounting for approximately $0.01-0.8 \%$ of dry weight (Van Agtmael et al., 1999). Chemically, artemisinin is a sesquiterpene trioxane lactone containing a peroxide bridge (Figure 4), which is essential for its activity (China Cooperative Research Group, 1982).

The earliest known record of A. annua was in the book 52 Prescriptions, discovered in the Mawangdui tomb of the Han Dynasty in $168 \mathrm{BC}$, where it was first described for the treatment of hemorrhoids (Qinghaosu Antimalarial Coordinating Research Group, 1979; Klayman, 1985). Around 340 AD, in Hong Ge's Handbook of Prescriptions for Emergency Treatment, a cold extraction method of qinghao was described for the treatment of intermittent fevers (Klayman, 1985). Qinghao has also been mentioned in several later standard Chinese Materia Medica texts as treatment for fever (Qinghaosu Antimalaria Coordinating Research Group, 1979; Hien and White, 1993); it has also been used for haemorrhoids, lice, wounds, boils, sores, and convulsions (Hsu, 2006).

The active ingredient of qinghao, artemisinin, was isolated from the plant $A$. annua in 1972 by a group of Chinese scientists (Hien and White, 1993). In 1975, its unique chemical structure was elucidated, as a sesquiterpene lactone bearing a peroxy group, which is believed to be responsible for the antimalarial activity of A. annua (Willcox et al., 2003), and is quite different from those of all known antimalarial drugs (Liu et al., 1979), then the name qinghaosu ("active principle of qinghao") was changed to artemisinin (Klayman, 1985). Its structure was determined by X-ray analysis in 1979 (Balint, 2001; Krishna et al., 2008). Further studies led to the development of more stable derivatives, such as dihydroartemisinin, artemether, artemotil (arteether) and artesunate (Ansari et al., 2013).

Artemisinin and its derivatives produce rapid parasite clearance, killing young circulating parasites more than the other forms of the parasite, before they are sequestered in the deep microvasculature (Dondorp and Von Seidlein, 2017). This phenomenon is important in the pathogenesis of malaria because mature parasites are able to adhere to endothelial cells, blood cells and platelets, which prevent their circulation in the bloodstream and they will therefore be able to escape clearance by the spleen (Buffet et al., 2011). This is clinically relevant, particularly in the cases of cerebral and severe malaria (Hsu, 2006). These drugs interact with heme to produce carbon-centered free radicals that alkylate protein and damage the microorganelles and membranes of the parasites (Meshnick, 1998), significantly faster than any other antimalarial does (Willcox et al., 2003; Sriram et al., 2004). Unlike the quinine-related drugs and antifolate drugs, artemisinin and its derivatives are also gametocytocidal and reduce the transmission of malaria by $P$. falciparum (Price et al., 1996).

Since 1994, artemisinins have been used in artemisinin-based combination therapies (ACTs) to treat uncomplicated malaria (Ouji et al., 2018), and by 2006, ACTs had become the recommended treatments for falciparum malaria worldwide (WHO, 2015). The artemisinin derivatives are usually combined with a more slowly eliminated drug in ACTs (Ansari et al., 2013). In this case, the role of the artemisinin compound is to reduce the number of parasites during the first 3 days of treatment (reduction of parasite biomass), while the role of the partner drug is to eliminate the remaining parasites leading to a malaria cure. ACTs are also recommended by WHO for chloroquine-resistant $P$. vivax malaria, and the injectable artesunate and artemether, are recommended for the treatment of severe malaria (WHO, 2018b).

ACTs are the most effective antimalarial medicines available today, but there are already cases of resistance to artemisinin and its derivatives. Artemisinin resistance in Cambodia was first identified in clinical studies in 2006, and few years later in Myanmar, Thailand, Viet Nam, and Lao. However, retrospective analysis of molecular markers indicated that artemisinin resistance probably emerged in 2001, before the widespread deployment of ACTs in Cambodia (Noedl et al., 2008; Dondorp et al., 2009; WHO, 2017). In spite of the fact that WHO continues to monitor cases of resistance to artemisinin and its derivatives, new drugs must be discovered to overcome the problem of the development of resistance that can make its use unfeasible in the future.

\section{Current Malaria Treatment}

ACTs are recommended by WHO as the first-and second-line treatment for uncomplicated P. falciparum malaria, as well as for chloroquine-resistant $P$. vivax malaria. The role of the artemisinin compound is to reduce the number of parasites during the first 3 days of treatment (reduction of parasite biomass), while the role of the partner drug is to eliminate the remaining parasites, leading to cure (WHO, 2018b). It is recommended to use a 3 -day treatment regimen with one of the five different ACTs, namely artemether/lumefantrine, artesunate/amodiaquine, dihydroartemisinin/piperaquine or artesunate/sulfadoxinepyrimethamine. ACTs are not recommended in the first trimester of pregnancy for uncomplicated $P$. falciparum malaria; the World Health Organization guidelines (WHO, 2015) recommends a combination of quinine and clindamycin, or only quinine for uncomplicated malaria caused by $P$. falciparum and by chloroquine-resistant $P$. vivax. 


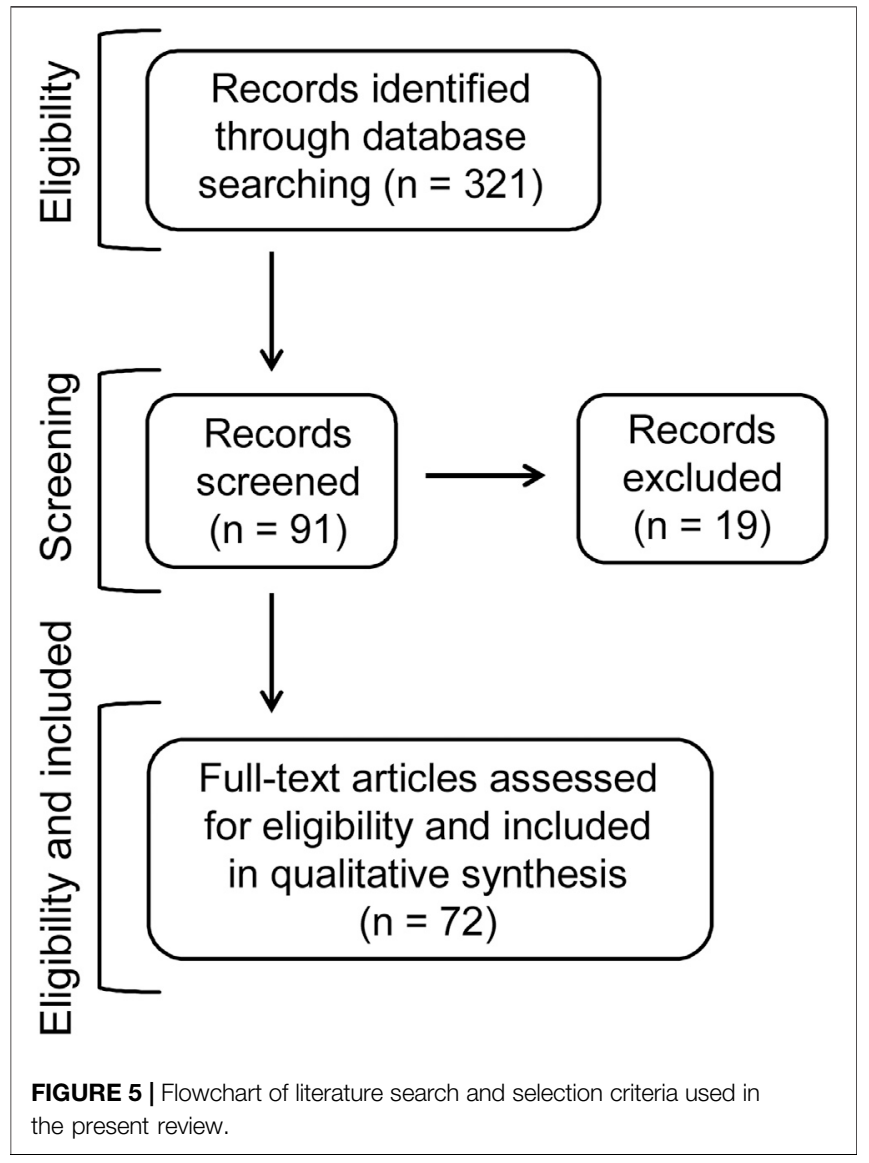

In areas with chloroquine-sensitive $P$. vivax, $P$. ovale, $P$. malariae or $P$. knowlesi infections, the adults and children are treated with ACTs or chloroquine. Primaquine can be administered to avoid relapses of vivax malaria in people with no Glucose-6-phosphate dehydrogenase deficiency. The treatment of adults and children with severe malaria is done with intravenous or intramuscular artesunate for at least $24 \mathrm{~h}$, but if parenteral artesunate is not available, intramuscular artemether is used in preference to quinine (WHO, 2015).

\section{METHODS}

\section{Study Selection and Analysis}

The flowchart (Figure 5) demonstrates the strategy used for identification, and the criteria for inclusion and exclusion of the plants focused in the present review. A total of 321 articles were initially retrieved from the database Pubmed in the last 10 years (2011-2020) using the keywords "antimalarials," "plants" and "natural products." Some additional references of other articles published before have been also included, although they were not found after using the keywords. In a further analysis, articles which focused on the plant species tested in vivo against malaria, using mice infected with Plasmodium species, were selected. This gave a total of 91 full articles, from which 19 articles were excluded because: 1 ) they were in other languages rather than English; 2) the reported chemosuppression was below $30 \%$, which did not correspond to the criteria for antimalarial activity, as defined below; 3) the tests were with polyherbal extracts rather than with one species of plant; 4) the active plants were toxic; 5) the tests against early sporogonic stages were conducted ex vivo; or, 6) the studies were performed using plants associated or in combination with antimalarial drugs. After applying all these requirements, the total number of publications evaluated was 72, as shown in Table 2 and Figure 5.

In this review natural products mostly from medicinal plants were considered active when inhibition of parasite growth was equal to or above $30 \%$ (the lower limit for moderately active products); those below $30 \%$ were considered to be inactive (Carvalho et al., 1991; Andrade-Neto et al., 2003; Coutinho et al., 2013; Induli et al., 2013; Musila et al., 2013; Nguta and Mbaria, 2013; Bantie et al., 2014; Upadhyay et al., 2014; Aguiar et al., 2015; Rocha e Silva et al., 2015; Satish et al., 2017).

\section{RESULTS AND DISCUSSION}

The scientific names of the plant species tested, their botanic families, parts of the plants fractionated, the ethnomedicinal uses and the countries where the studies were performed are shown in Table 2. Among the 72 articles, 54 of them evaluated the antimalarial activities of one plant species each while 18 of them evaluated two or three plant species each, giving rise to a total of 90 plant species, some of which were studied by more than one author. The three most studied families were Fabaceae, Apiaceae and Lamiaceae. The plant species evaluated for antimalarial activities corresponded to 44 botanical families; the parts of the plants tested were aerial parts, leaves, leaf latex, rhizomes, roots, root bark, stem bark, whole stem, branches, twigs, petiole, cortex, flower, fruits, fruit pulp, unripe fruits, and whole plant (Table 2).

A total of 60 of the 90 medicinal plant species (66.67\%) tested in mice with rodent Plasmodium species were recommended for the treatment of malaria and/or malaria prevention, as well as for other ailments; six species $(6.67 \%)$ were used against fever in general, which happens to be the main acute symptom of human malaria. A total of 15 species (16.67\%) had an ethnopharmacological recommendation for other diseases and morbidities rather than malaria, while nine species (10\%) did not have their ethnobotanical uses mentioned (Figure 6A).

Further information about the plant species studied are depicted in Table 2. Most studies regarding the antimalarial activities of plant species using animal models, were performed presumably where the plant species occur and/or are native: 59 of the species were in the African continent (65.56\%), 18 species were in Asia (20\%) and 13 species were in South America (14.44\%) (Figure 6B).

Regarding the countries where the studies were performed in mice infected with malaria parasites, an African country had the highest number, that is Ethiopia (Africa, $n=29$ ), followed by Brazil (South America, $n=11$ ), India (Asia, $n=10$ ) and Nigeria (Africa, $n=10$ ) (Figure 7). 
TABLE 2 | Botanical classification, ethnomedical uses of plants evaluated for antimalarial activity in mice infected with Plasmodium species, and countries of studies from 2011 to 2020.

\begin{tabular}{|c|c|c|c|c|c|}
\hline Family $^{a}$ & Plant species & Part(s) used & Ethnomedical use & Country & Reference \\
\hline \multirow[t]{2}{*}{ Acanthaceae (2) } & Acanthus polystachyus & Roots, leaves & Malaria and others & Ethiopia & $\begin{array}{l}\text { Derebe and Wubetu (2019), Kifle } \\
\text { and Atnafie (2020) }\end{array}$ \\
\hline & Hypoestes forskalei & Leaves & $\begin{array}{l}\text { Malaria, antipyretic, antileishmanial, } \\
\text { antitrypanosomal }\end{array}$ & Ethiopia & Misganaw et al. (2020) \\
\hline $\begin{array}{l}\text { Amaranthaceae } \\
\text { (1) }\end{array}$ & $\begin{array}{l}\text { Chenopodium } \\
\text { ambrosioides }\end{array}$ & Leaves & $\begin{array}{l}\text { Anti-inflammatory, anti-Leishmania and } \\
\text { others }\end{array}$ & Brazil & Cysne et al. (2016) \\
\hline \multirow[t]{2}{*}{ Anacardiaceae (2) } & Adansonia digitata & Stem bark & Malaria & Kenya & Musila et al. (2013) \\
\hline & Sorindeia juglandifolia & Fruits & Not found & Cameroon & Kamkumo et al. (2012) \\
\hline \multirow[t]{2}{*}{ Annonaceae (2) } & Polyalthia longifólia & Leaves & Malaria & Nigeria & Bankole et al. (2016) \\
\hline & Xylopia amazonica & Leaves, branches & Not found & Brazil & Lima et al. (2015) \\
\hline \multirow[t]{9}{*}{ Apiaceae (9) } & Daucus virgatus & Aerial parts & Not found & Tunisia & Sirignano et al. (2019) b \\
\hline & Ferulago angulata & Aerial parts & Sedative, tonic, and parasitic effects & Iran & Sajjadi et al. (2016) \\
\hline & Aspidosperma nitidum & $\begin{array}{l}\text { Wood bark, leaves, } \\
\text { branches }\end{array}$ & Malaria & Brazil & Coutinho et al. (2013) \\
\hline & $\begin{array}{l}\text { Aspidosperma } \\
\text { olivaceum }\end{array}$ & Stem bark, leaves & Fevers & Brazil & Chierrito et al. (2014) \\
\hline & Aspidosperma pyrifolium & Stem bark, stem & Inflammation process and dermatitis & Brazil & Ceravolo et al. (2018) \\
\hline & $\begin{array}{l}\text { Aspidosperma } \\
\text { ramiflorum }\end{array}$ & stem bark, leaves & Not found & Brazil & Aguiar et al. (2015) \\
\hline & Calotropis gigantea & Leaves, stems, flowers & Several non-antimalarial effects & Ethiopia & Satish et al. (2017) \\
\hline & Holarrhena pubescens & Roots & Malaria & Tanzania & Nondo et al. (2016) \\
\hline & Periploca linearifolia & Stem bark & Malaria & Ethiopia & Belay et al. (2018) \\
\hline Apocynaceae (1) & Acokanthera schimperi & Leaves & Malaria & Ethiopia & Mohammed et al. (2014) \\
\hline Arecaceae (1) & Euterpe oleracea & Fruit pulp & Anti-inflammatory and others effects & Brazil & Ferreira et al. (2019) \\
\hline \multirow[t]{4}{*}{ Asphodelaceae (4) } & Aloe $s p$ & Leaves & Malaria & Ethiopia & Mesfin et al. (2012) \\
\hline & Aloe pirottae & Leaves & Malaria & Ethiopia & Dibessa et al. (2020) \\
\hline & Aloe weloensis & Leaf latex & Malaria and several others diseases & Ethiopia & Teka et al. (2020) \\
\hline & Kniphofia foliosa & Rhizomes & Abdominal cramps and wound healing & Ethiopia & Induli et al. (2013) \\
\hline \multirow[t]{4}{*}{ Asteraceae (4) } & Dicoma tomentosa & Whole plant & Malaria & $\begin{array}{l}\text { Burkina } \\
\text { Faso }\end{array}$ & Jansen et al. (2012) \\
\hline & Echinops hoehnelii & Roots & Malaria & Ethiopia & Bitew et al. (2017) \\
\hline & Echinops Kebericho & Rizomes, roots & Malaria, fevers and others & Ethiopia & $\begin{array}{l}\text { Toma et al. (2015), Biruksew et al } \\
\text { (2018) }\end{array}$ \\
\hline & Helianthus annuus & $\begin{array}{l}\text { Roots, stems, seeds, } \\
\text { flowers, leaves }\end{array}$ & Malaria and others diseases & Indonesia & $\begin{array}{l}\text { Ekasari et al. (2019), Ekasari et al. } \\
(2020)\end{array}$ \\
\hline Bignoniaceae (1) & Markhamia tomentosa & Fresh leaves & Malaria & Nigeria & Bankole et al. (2016) \\
\hline Bombacaceae (1) & Bombax buonopozense & Root bark & Malaria, pain, fevers and diarrhoea & Nigeria & Christian et al. (2017) \\
\hline Boraginaceae (1) & Cordia africana & Leaves & Malaria & India & Wondafrash et al. (2019) \\
\hline \multirow[t]{2}{*}{ Combretaceae (2) } & Terminalia brownii & Barks & Malaria & Ethiopia & Biruk et al. (2020) \\
\hline & Terminalia macroptera & Leaves, roots & Malaria and others diseases & Mali & Haidara et al. (2018) \\
\hline \multirow[t]{3}{*}{ Cucurbitaceae (3) } & Citrullus colocynthis & Fruits & Malaria & Iran & Haddad et al. (2017) \\
\hline & Cucumis metuliferus & Leaves & Malaria & Tanzania & Mzena et al. (2018) \\
\hline & $\begin{array}{l}\text { Gynostemma } \\
\text { pentaphyllum }\end{array}$ & Leaves & Several diseases other than malaria & Thailand & Somsak et al. (2016) \\
\hline \multirow[t]{2}{*}{ Euphorbiaceae (2) } & Croton macrostachyus & Leaves & Malaria & Ethiopia & $\begin{array}{l}\text { Bantie et al. (2014), Mohammed } \\
\text { et al. (2014) }\end{array}$ \\
\hline & Phyllanthus nivosus & Leaves & $\begin{array}{l}\text { Malaria, fevers, headaches, toothaches, } \\
\text { tooth infections }\end{array}$ & Nigeria & Johnson et al. (2020) \\
\hline \multirow[t]{11}{*}{ Fabaceae (11) } & Acacia karroo & Leaves & Pyretic diseases & India & Sachdeva et al. (2020) \\
\hline & Acacia nilotica & Roots & Malaria & Nigeria & Alli et al. (2016) \\
\hline & Caesalpinia bonducella & Roots & Malaria & Tanzania & Nondo et al. (2016) \\
\hline & Caesalpinia pluviosa & Stem bark & Antiviral and other infections & Brazil & Kayano et al. (2011) \\
\hline & Commiphora africana & Stem bark & Malaria and other diseases & Tanzania & Kweyamba et al. (2019) \\
\hline & Copaifera reticulata & Oleoresin & Anti-inflammatory and other properties & Brazil & de Souza et al. (2017) \\
\hline & Dichrostachys cinérea & Stem bark, whole stem & Malaria and other diseases & Tanzania & Kweyamba et al. (2019) \\
\hline & Glycyrrhiza glabra & Roots & $\begin{array}{l}\text { To reduce the toxicity and enhances } \\
\text { effectiveness of other drugs }\end{array}$ & India & Kalani et al. (2013) \\
\hline & Indigofera spicata & Roots & Malaria & Ethiopia & Birru et al. (2017) \\
\hline & Pongamia pinnata & $\begin{array}{l}\text { Leaves, bark, flower, } \\
\text { root }\end{array}$ & $\begin{array}{l}\text { Antiplasmodial, antioxidant and other } \\
\text { effects }\end{array}$ & India & Satish and Sunita (2017) \\
\hline & Tamarindus indica & Fruits & Malaria & Ethiopia & Mesfin et al. (2012) \\
\hline \multirow{2}{*}{$\begin{array}{l}\text { Gentianaceae (1) } \\
\text { Icacinaceae (1) }\end{array}$} & Anthocleista djalonensis & Stem bark & Not found & Ivory Coast & Attemene et al. (2018) \\
\hline & Icacina senegalensis & Root bark & Antimalaria, antimicrobial and others & Nigeria & $\begin{array}{l}\text { Akuodor et al. (2017) } \\
\text { Continued on following page) }\end{array}$ \\
\hline
\end{tabular}


TABLE 2 | (Continued) Botanical classification, ethnomedical uses of plants evaluated for antimalarial activity in mice infected with Plasmodium species, and countries of studies from 2011 to 2020.

\begin{tabular}{|c|c|c|c|c|c|}
\hline Family $^{\mathbf{a}}$ & Plant species & Part(s) used & Ethnomedical use & Country & Reference \\
\hline \multirow[t]{7}{*}{ Lamiaceae (7) } & Ajuga bracteosa & Leaves & Malaria and other effects & India & Chandel and Bagai (2011) \\
\hline & Ajuga integrifólia & Aerial parts & Malaria & Ethiopia & Asnake et al. (2015) \\
\hline & Leonotis ocymifolia & Leaves & Malaria, yellow fever and others & Ethiopia & Teklu et al. (2020) \\
\hline & Ocimum lamifolium & Leaves & Malaria & Ethiopia & Kefe et al. (2016) \\
\hline & Ocimum sanctum & Leaves & To enhance immunity and others effects & India & Rajendran et al., 2014 \\
\hline & Ocimum suave & Leaves & Malaria & Kenya & Kiraithe et al. (2016) \\
\hline & Plectranthus barbatus & Root bark & Malaria & Kenya & Kiraithe et al. (2016) \\
\hline \multirow[t]{4}{*}{ Meliaceae (4) } & Azadirachta indica & Leaves & Malaria & Ethiopia & Mesfin et al. (2012) \\
\hline & $\begin{array}{l}\text { Entandrophragma } \\
\text { cylindricum }\end{array}$ & Stem bark & Malaria, yellow fever and other effects & Cameroon & Nadia et al. (2020) \\
\hline & Melia azedarach & Twigs & Malaria & Ethiopia & Asnake et al. (2015) \\
\hline & Trichilia heudelotii & Stem bark & Malaria & Nigeria & Bankole et al. (2016) \\
\hline Moraceae (1) & Ficus thonningii & Leaves & Several uses, non-antimalarial & Nigeria & Falade et al. (2014) \\
\hline Moringaceae (1) & Moringa oleifera & Leaves & Antioxidant and other effects & Thailand & Somsak et al. (2016) \\
\hline Myricaceae (1) & Myrica salicifolia & Roots & Malaria & Ethiopia & Kifle et al. (2020) \\
\hline \multirow[t]{2}{*}{ Myrtaceae (2) } & Psidium guajava & Leaves, unripe fruits & Broad spectrum of activities & India & Rajendran et al. (2014) \\
\hline & Syzygium cumini & Leaves & Pyretic diseases & India & Sachdeva et al. (2020) \\
\hline Ochnaceae (1) & Lophira alata & Leaves & Anti-fever and other uses & Nigeria & Falade et al. (2014) \\
\hline Oleaceae (1) & Olea europaea & Stem bark, leaves & Malaria and other infections & Ethiopia & $\begin{array}{l}\text { Misganaw et al. (2019), Hailesilase } \\
\text { et al. (2020) }\end{array}$ \\
\hline Picramniaceae (1) & Picramnia latifolia & Bark/petiole & Not found & Colombia & Berthi et al. (2018) \\
\hline Piperaceae (1) & Piper peltatum & Roots & Malaria & Brazil & Rocha e Silva et al. (2011) \\
\hline Poaceae (1) & $\begin{array}{l}\text { Andropogon } \\
\text { leucostachyus }\end{array}$ & Aerial parts & Malaria & Brazil & Lima et al. (2015) \\
\hline Ranunculaceae (1) & Coptis japônica & Rhizome & Inflammatory disease & Japan & Teklemichael et al. (2020) \\
\hline Rhamnaceae (1) & Ziziphus mauritiana & Leaves & Not found & Ivory Coast & Attemene et al. (2018) \\
\hline Rosaceae (1) & Rubus ellipticus & Leaves & Pyretic diseases & India & Sachdeva et al. (2020) \\
\hline \multirow[t]{3}{*}{ Rubiaceae (3) } & Canthium glaucum & Roots & Malaria & Kenya & Musila et al. (2013) \\
\hline & Gardenia ternifolia & Root bark & Malaria & Ethiopia & Nureye et al. (2018) \\
\hline & Heinsia crinita & $\begin{array}{l}\text { Leaves, fruits, stem } \\
\text { barks }\end{array}$ & Malaria, fever and others & Congo & Tshisekedi et al. (2017) \\
\hline \multirow[t]{3}{*}{ Rutaceae (3) } & Fagara zanthoxyloides & Leaves & Malaria & Nigeria & Enechi et al. (2019) \\
\hline & Murraya koenigii & Leaves & Malaria and to decrease insulin level & India & $\begin{array}{l}\text { Kamaraj et al. (2014), Rajendran } \\
\text { et al. (2014) }\end{array}$ \\
\hline & Zanthoxylum chalybeum & Root bark & Malaria & Kenya & Kiraithe et al. (2016) \\
\hline Sapindaceae (1) & Dodonaea angustifólia & Roots & Malaria & Ethiopia & Amelo et al. (2014) \\
\hline Santalaceae (1) & Osyris quadripartita & Leaves & Malaria & Ethiopia & Girma et al. (2015) \\
\hline Simaroubaceae(1) & Picrolemma huberi & Cortex & Not found & Colombia & Berthi et al. (2018) \\
\hline \multirow[t]{2}{*}{ Solanaceae (2) } & Physalis alkekengi & Leaves and fruits & Anti-fever and other uses & Iran & Haddad et al. (2017) \\
\hline & Solanum nigrum & Fruits & Not found & Iran & Haddad et al. (2017) \\
\hline Stilbaceae (1) & Nuxia congesta & Leaves & Malaria & Ethiopia & Fenta and Kahaliw (2019) \\
\hline Strychnaceae (1) & Strychnos mitis & Leaves & Malaria & Ethiopia & Fentahun et al. (2017) \\
\hline Tiliaceae (1) & Grewia trichocarpa & Roots & Malaria & Kenya & Mwangi et al. (2015) \\
\hline Verbenaceae (1) & Lippia kituiensis & Leaves & Malaria & Tanzania & Mzena et al. (2018) \\
\hline Zingiberaceae (1) & Zingiber officinale & Roots & Malaria & Ethiopia & Biruksew et al. (2018) \\
\hline Zygophyllaceae (1) & Balanites rotundifolia & Leaves & Malaria & Ethiopia & Asrade et al. (2017) \\
\hline
\end{tabular}

${ }^{a}$ Number of species studied by family in parentheses.

${ }^{b}$ Antimalarial activity evaluated against gametocytes.

Other details of the in vivo tests described in the original articles are summarized in Table 3. As expected, most medicinal plants exhibited antimalarial activities against Plasmodium species in mice experimentally infected $(P$. berghei or $P$. yoelli), though some active doses were too high. These doses are not feasible under clinical settings, making such extracts of no clinical relevance. The results validated the ethnobotanical uses of several plants in malaria endemic countries as they reduced parasitaemia and were well tolerated by the uninfected mice (Table 3 ).
The active doses of the extracts from medicinal plants tested in mice infected with rodent malaria species ranged from 1 to $6,000 \mathrm{mg} / \mathrm{kg}$, although there was no clear relationship between doses and percentage inhibition of parasite growth, in several cases. The mean survival time was assessed for $65(72.2 \%)$ of the species evaluated; for 55 of them (84.6\%), there was an increase in the survival time of the treated mice while for 10 plant species (15.4\%) there was no change in the time of animal survival, as compared to the untreated malaria controls (Table 3). The acute toxicity was evaluated for 40 of the 90 plant species; the $\mathrm{LD}_{50}$ values for 37 

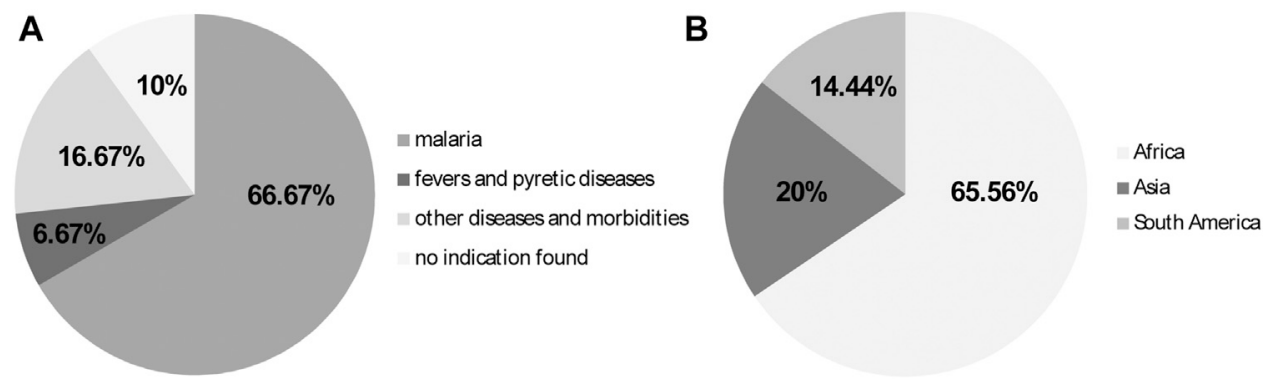

FIGURE 6 | (A) Ethnopharmacological uses of plant species evaluated against Plasmodium species in mice from 2011 to 2020 ; (B) Continents where tests were performed, presumably, where the plants occur or are natives.
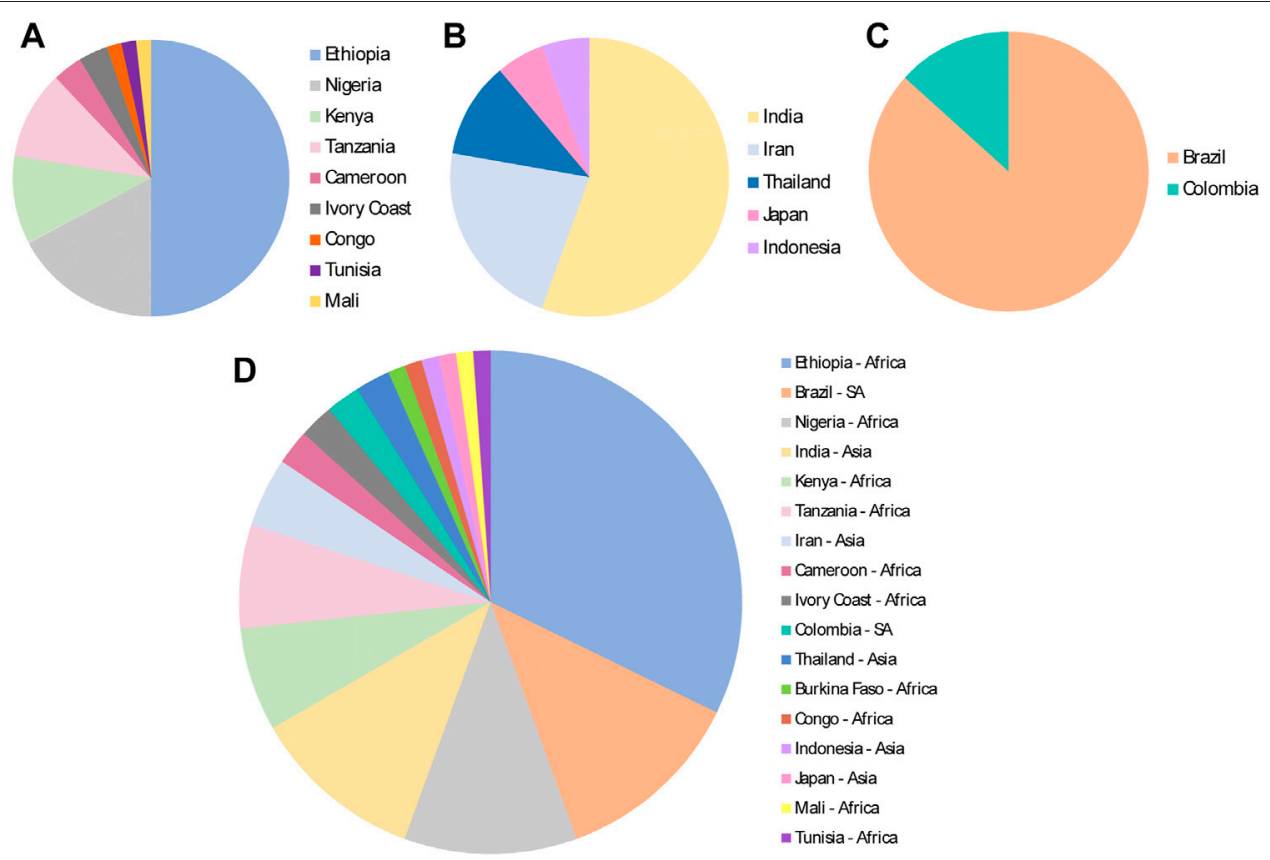

FIGURE 7 | Continents and countries where medicinal plants were evaluated for antimalarial activities in the murine malaria model, from 2011 to 2020 , based on total number of plant species evaluated. (A) Africa; (B) Asia; (C) South America (SA); (D) All different countries and regions.

species (41.1\%) were $2,000 \mathrm{mg} / \mathrm{kg}$ body weight or above (Table 3), and six of the plant species had $\mathrm{LD}_{50}$ values equal to or higher than $5,000 \mathrm{mg} / \mathrm{kg}$ body weight, suggesting that these plant extracts were not toxic (Lorke, 1983).

The most active species was Heliantus annuus (popularly know as sunflower) in tests using ethanolic extract of the leaves, which caused a high suppression of parasitemia (49, 76 , and $82 \%$ using oral doses of 1,10 and $100 \mathrm{mg} / \mathrm{kg}$, respectively). Its mechanism of action was through the in vitro inhibition of heme polarization by the parasites in a dose dependent manner. Extracts of other parts of the plant species, including roots, stems and flowers (Table 3) were also highly active (Ekasari et al., 2019; Ekasari et al., 2020), but the toxicity of the extracts was not evaluated, perhaps because the sunflower oil from seeds is largely used all over the world in food preparation.
Medicinal plants are often used in health care delivery as complementary and/or alternative medicines against human malaria, specially in poorer areas of the African countries. Such use may be important, especially in conjunction with other health measures like mosquito bed-nets, repellents, and/ or treatments with an antimalarial, including the inexpensive chloroquine pills, that costs about 10 US cents per course of treatment for an adult (Institute of Medicine, 2004).

The usefulness of traditional medicinal plants as antimalarials, as judged from the work herein analysed, is relevant, though some of the experimental work have not confirmed a dose response activity in the rodent malaria model, suggesting that they lack relevant activity. In addition and also importantly, in most studies organic solvents were used for the extraction of the plant materials tested, despite the fact that it has been recommended that only water and ethanol should be used in traditional preparations (Willcox et al., 2011). Therefore, 
TABLE 3 | Antimalarial activities and toxicities of medicinal plants evaluated in animal models from 2011 to 2020.

Plant species
Parasitemia Inhibition with each extract and dose used
the malaria infected mice (dose in $\mathrm{mg} / \mathrm{kg}$ body weight)

\author{
.
}

Plant species

Acacia karroo
Acacia nilotica
Acanthus polystachyus
Acokanthera schimperi
Adansonia digitata
Aloe sp
Aloe pirottae
Aloe weloensis
Ajuga bracteosa
Ajuga integrifólia
Andropogon
leucostachyus
Anthocleista djalonensis
Aspidosperma nitidum

Aspidosperma olivaceum

Aspidosperma pyrifolium

Aspidosperma ramiflorum

Azadirachta indica

Balanites rotundifolia

Bombax buonopozense

Caesalpinia bonducella

Caesalpinia pluviosa

Calotropis gigantea

Canthium glaucum

Chenopodium

ambrosioides

Citrullus colocynthis

Commiphora africana

Copaifera reticulata

Coptis japônica

Cordia africana

Croton macrostachyus

Cucumis metuliferus

Dichrostachys cinérea

Dicoma tomentosa

Dodonaea angustifólia

Daucus virgatus ${ }^{a}$

Echinops hoehnelii

Echinops kebericho

Entandrophragma

cylindricum

Euterpe oleracea

Fagara zanthoxyloides

Methanolic leaf extract 57\% (500)

Aqueous root fraction F-1 77\% (100)

Methanolic roots extract 33\% (200), 51\% (400) (Derebe and Wubetu, 2019);

methanolic leaves extract 34\% (200), 49\% (400) (Kifle and Atnafie, 2020)

Methanolic leaf extract 37\% (600)

Aqueous stem bark extract 60\% (100), organic stem bark extract 33\% (100)

Ethanolic leaf extract 74\% (650), aqueous leaf extract 58\% (650)

Aqueous latex extract 39\% (400), 47\% (600)

Aqueous leaf latex extract 42\% (200), 67\% (400)

Ethanolic leaf extract 67\% (250), 80\% (500), 85\% (750)

Methanolic aerial part extract 35\% (800)

Aqueous aerial part extract $71 \%(250)$

Hydroethanolic stem bark extract 36\% (200), 48\% (400), 71\% (600)

Ethanolic wood bark extract 48\% (250); chloroform fraction 43\% (250); methanolic extract >67\% (100); fraction from methanolic extract >48\% (100); FO III 66\% (50); FO IV 65\% (50); precipitate fraction 57\% (50)

Acidic stem bark fraction 79\% (100); 58\% (200)

Root bark extract 79\% (100), root extract 75\% (100); aqueous stem bark fraction $93 \%$ (100); alkaloid-rich stem fraction 79\% (100)

Methanolic stem bark neutral precipitate extract 66\% (250) and 53\% (500)

Ethanolic leaf extract 55\% (650)

Methanolic leaf extract 37\% (100), 42\% (200), 67\% (400)

Aqueous root bark extract $81 \%$ (50), 86\% (100), 93\% (200)

Dichloromethane root extract 38\% (400)

Ethanolic stem bark Fraction F4 79\% (50)

Methanolic leaf extract 41\% (100); 52\% (200); 65\% (400); 72\% (800); 74\% (1,000)

Aqueous root extract 32\% (100), organic extract 44\% (100)

Hydroalcoholic leaf extract 53\% (5)

Methanolic fruit extract 65\% (50)

Dichloromethane bark extract 64\% (400)

Oleoresin 96\% (100), 93\% (200)

Aqueous rhizome extract 50\% (122); coptisine chloride 89\% (365)

Methanol leaf extract $51 \%(600)$, butanol fraction $56 \%$ (400), chloroform fraction

45\% (400)

Methanol leaf extract 44\% (200), 78\% (400), 91\% (600); chloroform fraction 49\%

(200), 66\% (400), 76\% (600); methanol fraction 37\% (200), 53\% (400), 64\% (600); aqueous fraction 39\% (600) (Bantie et al., 2014); methanolic leaf extract 34\% (600), aqueous leaves extract 31\% (400), 51\% (600) (Mohammed et al., 2014)

Chloroform extract 46\% (300), 80\% (600), 99\% (1,500), methanolic extract 37\%

(300), 59\% (600), 89\% (1,500), ethyl acetate extract 31\% (300), 64\% (600), 84\% $(1,500)$

Dichloromethane bark extract 53\% (400)

Ethanolic 50\% whole plant extract 90\% (300); aqueous whole plant extract $~ 80 \%$

(300); methanol whole plant extract 40\% (100); methanolic + ethanolic 50\% extract $\sim 40 \%$ (100)

n-butanol root fraction 38\% (200), 56\% (400), 68\% (600); chloroform root fraction $37 \%$ (400), 42\% (600)

Methanolic aerial part Daucovirgolide G 92\% (50 $\mu \mathrm{g} / \mathrm{ml})$

Methanolic root extract 69\% (200), 79\% (400); dichloromethane fraction 34\% (200), $43 \%$ (400); fractons from 5-(penta-1, 3-diynyl)-2-(3,4-dihydroxybut-1-ynyl)-thiophene $43.2 \%$ (50) and 50\% (100); 5-(penta-1,3-diynyl)-2-(3-chloro-4-acetoxy-but-1-yn)thiophene $33 \%$ (100)

Ethanolic rhizome extract 58\% (500) (Toma et al., 2015); methanolic root extract 35\% Yes (500), 50\% (1,000) (Biruksew et al., 2018)

Ethyl acetate stem bark extract 99\% (250), 100\% (500)

Aqueous fruit pulp fraction 1 (total phenolics) $89 \%$ (20)

Methanolic leaf extract 82\% (200), 91\% (400), 96\% (600)
Safe dose to

non-infected mice $(\mathbf{m g} / \mathbf{k g}$

body weight)

survival of

the malaria

infected

mice

$\begin{array}{ll}\text { NE } & \text { NE } \\ \text { Yes } & \text { NE } \\ \text { Yes } & 2,000 \\ \text { Not improved } & 2,000 \\ \text { NE } & L D_{50}>1,000 \mu \mathrm{g} / \mathrm{ml} \\ \text { Not improved } & 3,000 \\ \text { Yes } & \text { NE } \\ \text { Yes } & 2,000 \\ \text { Yes } & \text { NE } \\ \text { Yes } & 2,000 \\ \text { Not improved } & \text { NE } \\ \text { Yes } & \\ \text { Yes } & \text { NE }\end{array}$

Not improved NE

NE NE

Yes NE

Not improved $\quad 3,000$

Yes 2,000

Yes $\quad 5,000$

Yes NE

NE NE

Yes NE

NE

Yes

$\mathrm{LD}_{50}>1,000 \mu \mathrm{g} / \mathrm{ml}$

NE

NE

NE

2,000

$\mathrm{NE}$

2,000

5,000 or 2,000

NE

NE

NE

2,000

NE

2,000

5,000

NE

$\begin{array}{ll}\text { Yes } & \text { NE } \\ \text { NE } & 5,000\end{array}$

(Continued on following page) 
TABLE 3 | (Continued) Antimalarial activities and toxicities of medicinal plants evaluated in animal models from 2011 to 2020.

Plant species
Parasitemia Inhibition with each extract and dose used
the malaria infected mice (dose in $\mathrm{mg} / \mathrm{kg}$ body weight)
Ferulago angulata

Ficus thonningii Gardenia ternifolia

Glycyrrhiza glabra Grewia trichocarpa Gynostemma pentaphyllum Heinsia crinita Helianthus annuus

Holarrhena pubescens Hypoestes forskalei Icacina senegalensis Indigofera spicata Kniphofia foliosa Leonotis ocymifolia Lippia kituiensis

Lophira alata Markhamia tomentosa Melia azedarach Moringa oleifera Murraya koenigii

Myrica salicifolia Nuxia congesta

Ocimum lamifolium Ocimum sanctum Ocimum suave Olea europaea

Osyris quadripartita Periploca linearifolia Phyllanthus nivosus Physalis alkekengi Picramnia latifolia Picrolemma huberi Piper peltatum Plectranthus barbatus Polyalthia longifólia Pongamia pinnata Psidium guajava

Rubus ellipticus Solanum nigrum Sorindeia juglandifolia

Strychnos mitis

Syzygium cumini Tamarindus indica Terminalia brownii
Ethanolic aerial part extract 30\% (100), 40\% (300), 50\% (600); superosin peroxidase 40\% (10), 50\% (30), 50\% (75), 75\% (100) Hexane leaf extracts 65\% (100), 71\% (200), 76\% (300), 83\% (400), 85\% (500) Yes Methanolic root bark extract 33\% (200), 47\% (400), 59\% (600); butanol fraction 31\% Yes (200), 42\% (400), 51\% (600); chloroform fraction 31\% (400), 41\% (600)

Butanolic root extract, 18ß-glycyrrhetinic acid 68-100\% (62.5-250)

Aqueous root extract 36\% (100)

Aqueous leaf extract 45\% (500), 50\% (1,000), 55\% (2,000)

Dichloromethane stem bark extract 49\% (300)

Ethanolic extracts: root 36\% (1), 48\% (10), 64\% (100), 72\% (250), 79\% (400), 63\% (800); stems 40\% (100); seeds 42\% (100); flowers 36\% (100) (Ekasari et al., 2019); leaf 49\% (1), 76\% (10), 82\% (100) (Ekasari et al., 2020)

Methanolic root extract 32\% (400), 43\% (800)

Methanolic leaf extract 47\% (200), 51\% (400), 56\% (600)

Ethanolic root extract 81\% (50), 86\% (100), 92\% (200)

Methanolic root extract 17\% (200), 35\% (400), 53\% (600)

Rhizome extract, Knipholone anthrone 30\% (100)

Hydroalcoholic extracts of leaf 7\% (100), 23\% (200), 37\% (400), 41\% (800)

Methanolic leaf extract 36\% (300), 69\% (600), 75\% (1,500); Chloroform leaf extract $46 \%$ (300), 70\% (600), 94\% (1,500); Ethyl acetate leaf extract 42\% (300), 70\% $(6,000), 95 \%(1,500)$

Hexane leaf extract 37\% (200), 60\% (300), 69\% (400), 74\% (500)

Aqueous leaf extract 46\% (250), 43\% (500), 73\% (800)

Methanolic aerial part extract $32 \%(800)$

Aqueous leaf extract 35\% (50), 40\% (100), 50\% (200)

Aqueous leaf extract 62\% (350), 72\% (750), 77\% (1,000); ethyl acetate leaf extract $87 \%$ (600); myristic acid 83\% (100); $\beta$-caryophyllene 88\% (100)

Methanolic root extract 38\% (100), 51\% (200), 59\% (400)

Hydromethanolic leaf extract 41\% (500), 45\% (750), 58\% (1,000); aqueous fraction 45\% (600)

Aqueous leaf extract 36\% (600)

Aqueous leaf extract 44\% (750), 36\% (1,000)

Chloroform-methanolic leaf extract 55\% (100)

Methanolic stem bark extract 30\% (200), 43\% (400), 52\% (600); butanol fraction 35\% (200), 45\% (400), compound C 38\% (200); methanolic leaf extract 50\% (200), 55\% (400), 58\% (600). Fractions: chloroform 32\% (200), 36\% (400), 38\% (600); butanol fraction $41 \%$ (200), 46\% (400), 51\% (600)

Chloroform leaf extract $41 \%(600)$

Methanolic stem bark extract 38\% (200), 43\% (400), 57\% (600)

Ethanolic leaf extract 83\% (100), 81\% (200)

Methanolic fruit and leaf extract 58\% (100)

Ethanolic bark/petiole extract 51\% $(1,000)$

Ethanolic cortex extract 93\% (150)

Chloroformic-ethanolic root extract, 4-Nerolidylcatechol 34\% (400), 63\% (600)

Chloroformic-methanolic root bark extract 79\% (100)

Aqueous leaf extract 53\% (800)

Methanolic bark extract $84 \%(1,000)$

Aqueous extract: unripe fruits 30\% (350), 65\% (750), 62\% (1,000); leaves 74\% (350), $80 \%$ (750), 86\% (1,000)

Methanolic seed extract $64 \%(500)$

Methanolic fruit extract $61 \%$ (100)

Methanolic fruit extract, compound 1 - 2,3,6-trihydroxy benzoic acid (1) 54\% (50), $70 \%(100)$

Aqueous leaf extract 75\% (400), 96\% (600); hydro-methanolic leaf extract 36\% (200), Yes $81 \%$ (400), 94\% (600)

Methanolic leaf extract $52 \%(500)$

Aqueous fruits extract $81 \%(650)$

Methanol bark extract 33\% (100), 47\% (200), 60\% (400); aqueous bark extract 39\% (200), 51\% (400)

\section{Increased survival of the malaria infected \\ mice}

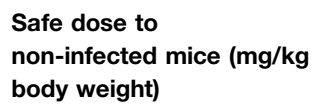

Safe dose to non-infected mice $(\mathrm{mg} / \mathbf{k g}$ body weight)

Yes

NE

$\mathrm{NE}$

2,000

NE NE

Not improved $\quad L_{50}$ of $545.8 \mu \mathrm{g} / \mathrm{ml}$

NE $\quad 4,000$

NE NE

Yes NE

Not improved NE

Yes 2,000

Yes NE

Yes NE

NE NE

Not improved 2,000

Yes NE

NE

$\mathrm{NE}$

NE

2,000

4,000

$\mathrm{NE}$

2,000

5,000

2,000

$\mathrm{NE}$

2,000

2,000

$\begin{array}{ll}\text { NE } & 2,000 \\ \text { Yes } & 2,000\end{array}$

Yes 2,000

Yes 2,000

NE NE

Yes NE

NE 2,000

NE $\quad 2,000$

Yes NE

NE $\quad 2,000$

NE NE

Yes $\quad$ NE

$\mathrm{NE}$
$\mathrm{NE}$

NE

NE

7,000

2,000

NE NE

Not improved $\quad 3,000$

2,000

(Continued on following page) 
TABLE 3 | (Continued) Antimalarial activities and toxicities of medicinal plants evaluated in animal models from 2011 to 2020.

\begin{tabular}{|c|c|c|c|}
\hline Plant species & $\begin{array}{l}\text { Parasitemia Inhibition with each extract and dose used for the treatment of } \\
\text { the malaria infected mice (dose in } \mathrm{mg} / \mathrm{kg} \text { body weight) }\end{array}$ & $\begin{array}{l}\text { Increased } \\
\text { survival of } \\
\text { the malaria } \\
\text { infected } \\
\text { mice }\end{array}$ & $\begin{array}{l}\text { Safe dose to } \\
\text { non-infected mice }(\mathrm{mg} / \mathrm{kg} \\
\text { body weight) }\end{array}$ \\
\hline Terminalia macroptera & Leaves ethanolic extract 37\% (100), roots ethanolic extract 46\% (100) & Not improved & 2,000 \\
\hline Trichilia heudelotii & Aqueous stem bark extract 40\% (800) & $\mathrm{NE}$ & $\mathrm{NE}$ \\
\hline Xylopia amazônica & Aqueous leaf and branch extract $52 \%$ (250) & Not improved & NE \\
\hline Zanthoxylum chalybeum & Aqueous leaf extract $78 \%(100)$ & NE & 2,000 \\
\hline Zingiber officinale & Methanolic root extract 33\% $(1,000)$ & Yes & NE \\
\hline Ziziphus mauritiana & Hydroethanolic stem bark extract 39\% (200), 66\% (400), 89\% (600) & Yes & $\mathrm{NE}$ \\
\hline
\end{tabular}

NE, not evaluated.

${ }^{a}$ Antimalarial activity evaluated in gametocytes.

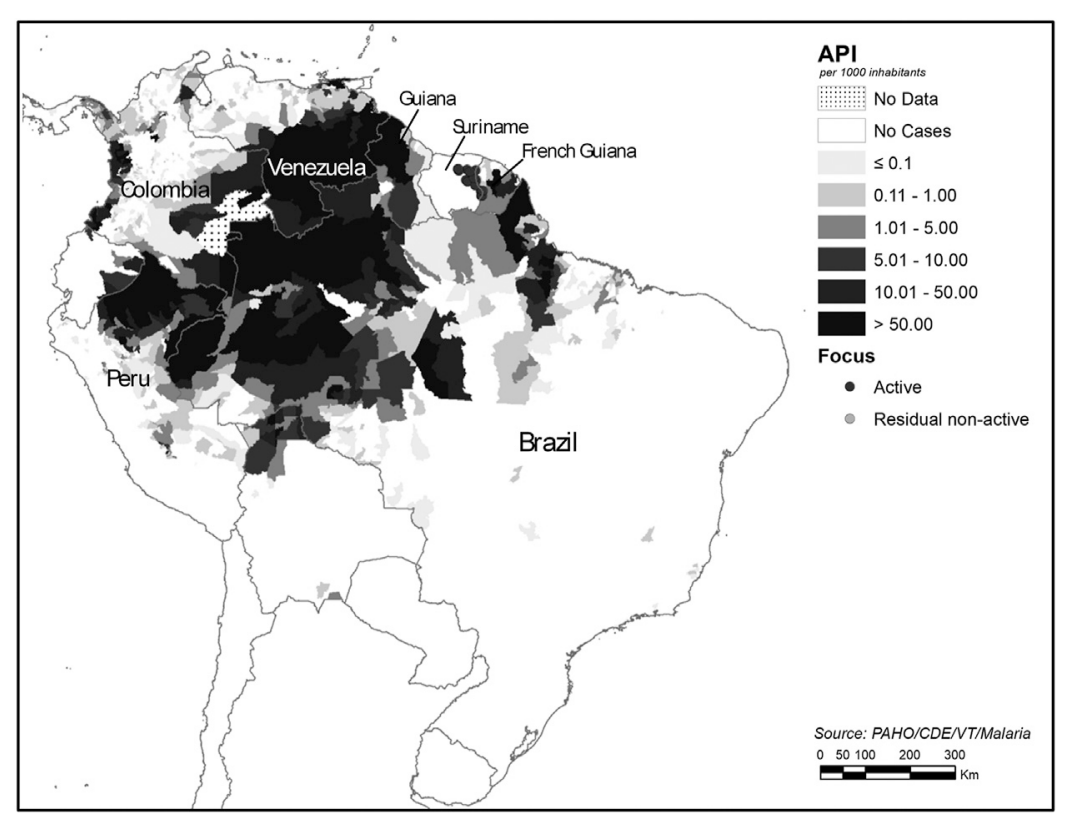

FIGURE 8| Malaria by Annual Parasite Index (AP1) in the Americas (Pan American Health Organization, 2017), adapted by the authors. Source: https://www.paho. org/hq/index.php?option=com_topics\&view=article\&id=33\&ltemid=40757\&lang=en.

with the present analysed data, it is difficult to evaluate the real pharmacological usefulness of medicinal plant extracted in organic solvents to serve as future bioproduct for the development of antimalarials.

\section{Tests of Medicinal Plants From Brazil Against Experimental Rodent Malaria}

In Brazil, plants and other natural products are rarely used to treat or prevent malaria. This, in part, is due to the fact that the Public Health system provides diagnosis and treatment of the disease free of charge. The specific diagnosis is promptly provided by the public health system of the Ministry of Health in Brazil, followed by treatment with antimalarials. In addition, human malaria is of compulsory notification to the Public Health system, that also controls the antimalarial drug distribution, under the
State governments in Brazil (Ministério, 2020). Treatment is only provided after the parasite species has been parasitologically confirmed, and it varies according to the diagnosis of the species of parasite, which at present is mainly $P$. vivax followed by $P$. falciparum. The malaria endemic areas are restricted to the Amazon region in Brazil and its neighbouring countries in South America: Colombia, Peru and Venezuela and the Guyanas (Figure 8).

In the Amazon region, the Quilombolas are endowed with extensive experience in the use of medicinal plants, as they have centuries of close contact with and dependence on local biodiversity as a means of livelihood and therapeutic resources. This fact makes the traditional communities attractive for groups conducting ethno-directed studies for medicinal plants used against malaria and other diseases (Oliveira et al., 2015). The popular uses of one species of 
medicinal plant, Ampelozyzyphus amazonicus, known as "Indian beer," has been previously described, and found to be frequent among the indigenous people in the State of Amazon (Botelho et al., 1981; Brandão et al., 1985; Carvalho et al., 1991; Brandão et al., 1992; Krettli et al., 2001; Oliveira et al., 2011), and also common among the Quilombolas (Oliveira et al., 2011, 2015).

It has been demonstrated that the ethanolic extracts of $A$. amazonicus target the sporozoites, the infective form inoculated through the mosquito bite; in vitro and in vivo models were used in the study (Andrade-Neto et al., 2008). The animals treated with ethanolic extracts by oral route, prior to sporozoite inoculation intravenously, had a significantly prolonged malaria pre-patent period (which is the time relapsed between sporozoite inoculation and detection of parasitemia in the experimental animals). The treated mice had a lower parasitemia and a prolonged survival time, as compared to the untreated control mice infected with sporozoites. Additional in vitro tests clearly confirmed the in vivo results: sporozoites pre-incubated with the plant extracts, were less infective to host cells, as compared to control sporozoites in culture medium. In conclusion, the "Indian beer" ethanolic extract was shown to be a potent prophylactic against malaria (Andrade-Neto et al., 2008). In other studies, it was clearly shown that the extracts of "Indian beer" were inactive against the blood forms of the parasite in mice (Botelho et al., 1981; Brandão et al., 1992).

Unfortunately, very few groups studying the experimental activity of medicinal plants in the world have facilities to produce sporozoites for the in vivo and in vitro tests. Thus, compounds active against these forms, or against the intrahepatic parasites developed from sporozoites will not be discovered until such tests are more easily performed with medicinal plants aiming at new antimalarials. Our group has developed an experimental model to test extracts from plants against the sporogonic stages in mosquitoes as well as the tissue cycle of sporozoite development, using an avian malaria parasite $P$. gallinaceum and mosquitoes Aedes, which are susceptible to the species (Carvalho et al., 1992).

Another medicinal plant species frequently used to treat fevers and malaria in Brazil is Bidens pilosa, which is active against malaria and was listed as an antimalarial medicinal plant of interest to the Unified Health System in Brazil, largely based on experimental studies of our group (Andrade-Neto et al., 2004; Oliveira et al., 2004; Palhares et al., 2015). The ethanolic crude extracts from $B$. pilosa caused $60 \%$ reduction in parasitaemia at doses of $250 \mathrm{mg} / \mathrm{kg}$ in mice infected with $P$. berghei; importantly, the ethanolic extracts from B. pilosa were similarly active in vitro against $P$. falciparum chloroquine-resistant (clone W2) and chloroquine-sensitive parasites (clone D6) (Andrade-Neto et al., 2004).

Essential oils (EOs) have been considered as an important class of antimalarial natural products with low molecular weight components, rich in monoterpenes and sesquiterpenes, found specially in plants native to Northeast Brazil (Mota et al., 2012). Thus, EOs obtained from Vanillosmopsis arborea (Asteraceae) were active sub-cutaneously, causing up to $47 \%$ inhibition of parasitemia in mice. EOs present in Lippia sidoides Cham. (Verbenaceae) and in Croton zehntneri (Euphorbiaceae) were active by oral route, causing $43-55 \%$ malaria growth inhibition, respectively, although no dose response activity was observed. The active EOs had monoterpene and phenylpropanoid compounds like estragole, $\boldsymbol{\alpha}$-bisabolol and thymol active in vitro against $P$. falciparum but not tested in mice with malaria (Mota et al., 2012). Another plant that contains an oleoresin rich in sesquiterpenes and diterpenes, but with high in vivo activity, is Copaifera reticulata, a tree distributed throughout the Amazon region, which has $\beta$-caryophyllene as its major compound, and caused a reduction of $93 \%$ in parasitemia when it was tested in mice (de Souza et al., 2017).

A high antimalarial activity has been described in the extracts of another 11 Brazilian medicinal plants, namely, Chenopodium ambrosioides (Cysne et al., 2016), Xylopia amazonica (Lima et al., 2015), Aspidosperma nitidum (Coutinho et al., 2013), A. olivaceum (Chierrito et al., 2014), A. pyrifolium (Ceravolo et al., 2018), A. ramiflorum (Aguiar et al., 2015), Euterpe oleracea (Ferreira et al., 2019), Caesalpinia pluviosa (Kayano et al., 2011), Copaifera reticulata (de Souza et al., 2017), Piper peltatum (Rocha e Silva et al., 2011) and Andropogon leucostachyus (Lima et al., 2015); all of them have been described as active against experimental malaria in mice and also in vitro against $P$. falciparum. The cited genus Aspidosperma spp, was the most studied among the Brazilian plants in the last 10 years, and has been considered for further studies in drug development; the species A. pyrifolium (at $100 \mathrm{mg} / \mathrm{kg}$ ) and $A$. olivaceum (at 100 and $200 \mathrm{mg} / \mathrm{kg}$ ) were the most potent in vivo against $P$. berghei murine malaria, with a parasitemia reduction of 75-93\% for A. pyrifolium (Ceravolo et al., 2018), and 58 and $79 \%$ for A. olivaceum respectively (Chierrito et al., 2014) (Tables 1, 2).

\section{Natural Products Isolated From Medicinal Plants and Their Activities}

Of all the antimalarial medicinal plants studied from 2011 to 2020, very few have their active principles isolated and characterized, such as Coptis japonica, Heinsia crinata, Piper peltatum and Murraya koenigii. Some of the active principles have been previously isolated from other plants while others are novel. Most of the studies carried out on the active principles are mainly in vitro studies. This is partly due to the fact that these compounds were isolated in small amounts that allowed only in vitro studies, not a main focus of this review. To overcome such bottle-neck, it is important to start the process of isolation of compounds using large amount of plant materials. The isolated compounds evaluated for in vivo antimalarial activities were: 1) Coptisine Chloride from Coptis japonica, (Teklemichael et al., 2020); 2) 4-Nerolidylcatechol isolated from Piper peltatum (Rocha e Silva et al., 2011); and 3) Myristic Acid and $\beta$-Caryophyllene from Murraya koenigii (Kamaraj et al., 2014) (Tables 2, 3).

In the studies with Piper peltatum, very high doses of 4Nerolidylcatechol, the active principle $(400$ and $600 \mathrm{mg} / \mathrm{kg}$ body weight), have been tested, resulting in chemosuppression of 34.4 and $63.1 \%$, respectively, in P. berghei NK65-infected mice (Rocha e Silva et al., 2011). Such high doses are not clinically 
TABLE 4 | Acute toxicities of antimalarial compounds isolated from medicinal plants.

\begin{tabular}{|c|c|c|c|c|c|}
\hline Plant species & $\begin{array}{l}\text { Names of } \\
\text { compounds }\end{array}$ & Animal & Route & $\mathrm{LD}_{50}$ (mg/kg body weight) & References \\
\hline \multirow[t]{5}{*}{ Coptis japonica } & Palmatine & Mouse & Oral & $1,533.68$ & Yi et al. (2013) \\
\hline & Berberine & Mouse & Intraperitoneal & 57.6103 & Kheir et al. (2010) \\
\hline & & & Oral & 713.57 & Yi et al. (2013) \\
\hline & Coptisine & Mouse & Oral & 852.12 & Yi et al. (2013) \\
\hline & & & Intravenous & 9.0386 & Kheir et al. (2010) \\
\hline Murraya koenigii & Myristic Acid & Mouse & Intravenous & 43 & Oro and Wretlind (1961) \\
\hline Piper peltatum & 4-Nerolidylcatechol & Mouse & Oral & 673.22 & Mendanha da Cunha et al. (2013) \\
\hline
\end{tabular}

feasible for human use; thus, efforts should be directed towards the development of compounds with higher activities at low doses as antimalarial drugs.

The 4-day suppressive test (Peters, 1965) was used for all the in vivo studies. However, one of the studies failed to determine the parasitaemia of day 4 post-inoculation, which is very important for identifying the fast acting compounds against malaria. It is only the slow acting compounds that are left till days 5, 6, and 7 post-inoculation (Fidock et al., 2004).

One of the compounds, Coptisine Chloride, was administered intraperitoneally, which is not the conventional route of administration for in vivo studies in murine malaria. The pharmacokinetic parameters of the drugs administered via such route are different from those of conventionally used routes of administration and therefore, they are not applicable to humans (Teklemichael et al., 2020).

Some pure compounds such as Aspidoscarpine, Uleine, Apparicine, and N-Methyl-Tetrahydrolivacine were isolated from A. olivaceum (Chierrito et al., 2014), Isositrikine, 10Methoxygeissoschizol and Ramiflorine were isolated from $A$. ramiflorum (Aguiar et al., 2015), and the bisindole alkaloid Leucoridine B was isolated from A. pyrifolium (Ceravolo et al., 2018); but none of these pure compounds was tested in the experimental mouse model. Braznitidumine was isolated from $A$. nitidum (Coutinho et al., 2013), but it was not active against $P$. berghei in experimentally infected mice.

Only one compound was tested against the life cycle in the mosquito, namely, Daucovirgolide G extracted from the plant Daucus virgatus; it is impressive that it inhibits $92 \%$ of the early sporogonic stages of the parasite at $50 \mu \mathrm{g} / \mathrm{ml}$ (Sirignano et al., 2019; Table 4). This transmission blocking activity appears to be related to the presence of an intact endocyclic double bond system of the compound, that interacts with the biological target (Sirignano et al., 2019). This structural part is obviously lacking in Daucovirgolide J, which is the reason for its lack of activity (Sirignano et al., 2019).

Africa has a rich flora, ranging from the Savannah to the rainforest, with diversity of phytochemicals and other biomolecules which possess various pharmacological activities, some of which have been extensively reviewed elsewhere (Moyo et al., 2015; Van Wyk, 2015; Ahmed et al., 2018; Oguntibeju, 2018; Van Vuuren and Frank, 2020). Many plants have been reported to be used for the treatment of malaria in the African continent (Soh and Benoit-Vical, 2007; Pillay et al., 2008; Adebayo and Krettli,
2011; Maranz, 2012; Chinsembu, 2015; Memvanga et al., 2015; Omara et al., 2020). As the use of Cinchona species (from which quinine was isolated) for the treatment of malaria dates back to the 1700s (Guerra, 1977; Lee, 2002), the use of some plants, such as Azadiractha indica and Alstonia broonei, for the treatment of malaria, indigenously, in the African continent dates back to centuries, though this has not been properly documented. More recently, the efficacies of some of these medicinal plants have been scientifically authenticated and their active principles isolated, with the mechanisms of action of some of them delineated. However, none of the isolated compounds has been developed into drugs to be used clinically for the treatment of malaria, as reviewed below.

The mechanisms of action of most isolated compounds from the medicinal plants are unclear and/or were only studied in vitro against $P$. falciparum. This is the case of Coptisine, isolated from Coptis japonica, which exhibits its activity by inhibiting $P$. falciparum dihydroorotate dehydrogenase, an enzyme required for the synthesis of pyrimidine in the parasite (Lang et al., 2018). The authors reported that the kinetic parameters obtained revealed that coptisine was an uncompetitive inhibitor of the enzyme. Berberine, isolated from Coptis japonica, has been shown to inhibit telomerase activity in P. falciparum (Sriwilaijareon et al., 2002), but there are no documents of studies with their activities in the malaria infected mice.

The chemical structure and molecular formula of each isolated compound are shown in Table 5.

\section{Acute and Sub-Chronic Toxicity of Some Isolated Compounds}

Some of the compounds isolated from Coptis japonica (Teklemichael et al., 2020), Piper peltatum (Rocha e Silva et al., 2011) and Murraya koenigii (Kamaraj et al., 2014) plants have been evaluated for their acute toxicities (Table 4). None of them could be declared safe, having oral $\mathrm{LD}_{50}$ values that are less than 5,000 mg/kg body weight (Lorke, 1983). Among the compounds evaluated for acute toxicity, Palmatine was the least toxic with the highest oral $\mathrm{LD}_{50}$ value. However, many of the isolated compounds have not been evaluated for their acute toxicity. This may also be due to fact that these compounds were isolated in small amounts which were not sufficient enough to allow their being tested for acute toxicity. Other compounds were tested by intraperitoneal route 
TABLE 5 | Some antimalarial compounds isolated from medicinal plants.

Isolated compound

Coptisine chloride

Molecular formula

$\mathrm{C}_{19} \mathrm{H}_{14} \mathrm{ClNO}_{4}$

4-Nerolidylcatechol

$\mathrm{C}_{21} \mathrm{H}_{30} \mathrm{O}_{2}$

Myristic acid

$\mathrm{C}_{14} \mathrm{H}_{28} \mathrm{O}_{2}$

$\beta$-Caryophyllene

$\mathrm{C}_{15} \mathrm{H}_{24}$

Aspidoscarpine

$\mathrm{C}_{11} \mathrm{H}_{18} \mathrm{~N}$

Uleine

$\mathrm{C}_{18} \mathrm{H}_{22} \mathrm{~N}_{2}$

$\mathrm{C}_{18} \mathrm{H}_{20} \mathrm{~N}_{2}$

Apparicine

Isositrikine
$\mathrm{C}_{18} \mathrm{H}_{20} \mathrm{~N}_{2}$

$\mathrm{C}_{21} \mathrm{H}_{26} \mathrm{~N}_{2} \mathrm{O}_{3}$

\section{Chemical structure}<smiles>ClC1=Cc2c3ccc(CCN4C5=CC6=C(C=C4CC5)OCO6)c2C1=C3</smiles><smiles>C=CC(C)(CC/C=C(\C)CCC=C(C)C)c1ccc(O)c(O)c1</smiles>

HO<smiles>CCCCCCCCCCCCCC(=O)O</smiles><smiles>C=C1CC/C=C(/C)CC[C@@]2(F)C[C@H]1CC2(C)C</smiles><smiles>CCC12CCCC3NCCC34Cc3ccc(OC)c(O)c3N(C(C)=O)[C@H]1CC[C@@]42C</smiles><smiles>C=C1c2[nH]c3ccccc3c2C2C(CC)C1CN2C</smiles><smiles>C=C1CN2CCC(C1=C)c1[nH]c3ccccc3c1C2</smiles><smiles>Cc1c2c(cc3c1[nH]c1ccccc13)C(C)N(C)CC2</smiles><smiles>C/C=C1/CC2CCc3c([nH]c4ccccc34)C2CC1C(CO)(CO)C(=O)OC</smiles>

(Continued on following page) 
TABLE 5 | (Continued) Some antimalarial compounds isolated from medicinal plants.

\section{Isolated compound}

10-Methoxygeissoschizol

Ramiflorine $\mathrm{A}$ and $\mathrm{B}$

$\mathrm{C}_{30} \mathrm{H}_{34} \mathrm{~N}_{4} \mathrm{O}$

$\mathrm{C}_{38} \mathrm{H}_{42} \mathrm{~N}_{4}$

Leucoridine B

Braznitidumine

Daucovirgolide G

$\mathrm{C}_{25} \mathrm{H}_{34} \mathrm{O}_{7}$

$\mathrm{C}_{20} \mathrm{H}_{18} \mathrm{NO}_{4}$

\section{Chemical structure}

$\mathrm{C}_{20} \mathrm{H}_{26} \mathrm{~N}_{2} \mathrm{O}_{2}$
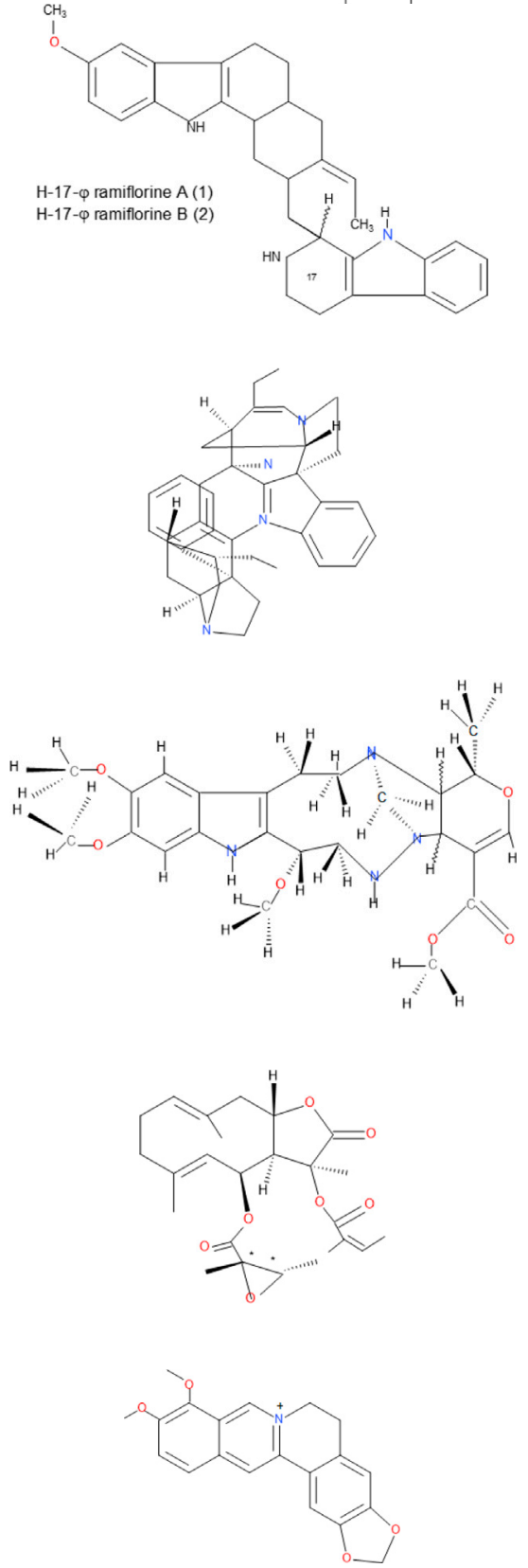

(Berberine) or by intravenous route (Myristic Acid) which are not routes used traditionally for the treatment of malaria in humans.
The subchronic systemic toxicity, defined as adverse effects occurring after the repeated or continuous administration of a test sample for up to 90 days, does not 
seem to be related to the extracts that have been used against malaria; furthermore, only Coptisine Chloride was tested in vivo (Teklemichael et al., 2020) (Table 3). Other studies have shown that administration of Palmatine, Coptisine and Berberine, at the dose of $156 \mathrm{mg} / \mathrm{kg}$ body weight, did not exert nephrotoxic or hepatotoxic effects, though Palmatine significantly increased $(p<0.05)$ plasma total bilirubin concentration compared to control (Yi et al., 2013). However, the authors did not proceed further to identify whether hemolysis, impaired conjugation of bilirubin in the liver or excretion of conjugated bilirubin into the bile duct was responsible for such increase. Many of the compounds have not been evaluated for sub-chronic toxicity or for chronic toxicity. This has limited the determination of the no adverse effect levels (NOAEL) and low adverse effect levels (LOAEL) of the compounds.

\section{Genotoxicity}

Berberine, a compound isolated from Coptis japonica (Table 4), has been reported not to be genotoxic for prokaryotic cells (Pasqual et al., 1993); however, it has been reported to be genotoxic to dividing eukaryotic cells by intercalating with the DNA (Jennings and Ridler, 1983). Palmatine has been reported to exert genotoxicity causing DNA double strand breaks, though to a lesser extent than Berberine (Chen et al., 2013). Both compounds have been reported to inhibit topoisomerase I and II activities, thereby inhibiting DNA relaxation and decatenation during replication (Chen et al., 2013). Inhibition of DNA decatenation results in the stabilization of topo II-DNA complexes ( $\mathrm{Li}$ et al., 2010), thereby enhancing the induction of DNA double strand breaks. Palmatine has been reported to interact with DNA by binding to the groove of DNA ( $\mathrm{Mi}$ et al., 2015). Knipholone and knipholone anthrone have been reported not to cause DNA damage on their own but knipholone anthrone caused DNA damage in the presence of copper ions through the generation of hydrogen peroxide (Habtemariam and Dagne, 2009). 4-Nerolidylcatechol has been shown to exhibit low genotoxicity compared to negative control. However, 4-Nerolidylcatechol has been reported to protect against cyclophosphamide-induced DNA damage by scavenging the free radicals generated by cyclophosphamide (Valadares et al., 2007).

\section{Mutagenicity}

It has been reported that berberine, in the absence of microsomal fraction S9, was weakly mutagenic in Salmonella typhimurium TA98, a frameshift detecting strain (Nozaka et al., 1990). It did not cause an increase in the frequency of point mutation (Pasqual et al., 1993) under conditions in which the frameshift mutation was increased by a factor of 4 in Saccharomyces cerevisiae using this model (Pasqual et al., 1993). None of the studies evaluated the mutagenic activities of the compounds using the murine malaria parasite model which analyses the micronucleated cells (Tometsko et al., 1993).

\section{Carcinogenicity, Reproductive Toxicity and Allergenicity}

Several purified compounds exert carcinogenic effects after accumulation, over time, in cellular systems. The searches made on PubMed and PubChem revealed that the above isolated compounds have not been evaluated for carcinogenic and allergenic effects. Their effects on pregnancy, such as absorption of fetus, and teratogenic effects have not been evaluated. This indicates that all the isolated compounds have not fully gone through the process of drug development. Consequently, not a single one of them has been developed into an antimalarial drug, neither been subjected to clinical trials.

\section{CONCLUSION AND FUTURE PERSPECTIVES}

Of the seventy one manuscripts published in the past 10 years that evaluated the antimalarial activities of extracts and isolated compounds from plant species in rodent malaria model, most of them aimed the erythrocytic stages of the parasite, which are responsible for the malaria symptoms. Only one compound was tested against the early sporogonic stages of the parasite. In addition, a few active principles were described. All steps of extractions, from the pre-extraction to final extraction are equally important in the study of medicinal plants, and play a critical role in the outcomes, including the product yield and phytochemical characterization; and these steps seem to interfere in the final product activity (Azwanida, 2015). Most of the experimental studies of plant materials used organic solvents, in spite of the recommendation in the specific literature that only water and ethanol should be used in traditional preparations for medical use (Willcox et al., 2011). Taken together, these facts explain the reduced contribution of Africa, in the past 10 years, to the achievement of the Medicine for Malaria Venture objective, which is the development of a new antimalarial drug every 5 years. The reason for this is not far-fetched but in part, it is due to the gross lack of standard facilities for antimalarial drug development in various countries of the African continent, which is responsible for most of the antimalarial tests herein described, especially in countries like Ethiopia and Nigeria, where most of the results have been published in the last 10 years. It is believed that technological advancement in the African continent and the world at large, will certainly go a long way in tackling this fundamental problem, thereby liberating the continent from the scourge of this disease.

\section{AUTHOR CONTRIBUTIONS}

IC designed the figures and prepared the tables; AA performed the bibliographic survey of the articles; JA wrote about plant isolated compounds, their toxicity, genotoxicity, mutagenicity and carcinogenicity; AK supervised the work and critically reviewed the manuscript. All the authors contributed to writing and analysing the paper. 


\section{FUNDING}

This work was supported by a grant from the Conselho Nacional de Desenvolvimento Científico e Tecnológico (CNPq) 409751/2018-9, and FASESP 2019/19708-0, Brazil. AK is a recipient of a Senior Fellowship from CNPQ, Brazil.

\section{REFERENCES}

Abay, S. M., Lucantoni, L., Dahiya, N., Dori, G., Dembo, E. G., and Esposito, F. (2015). Plasmodium Transmission Blocking Activities of Vernonia Amygdalina Extracts and Isolated Compounds. Malar. J. 14, 288. doi:10.1186/s12936-0150812-2

Achan, J., Talisuna, A. O., Erhart, A., Yeka, A., Tibenderana, J. K., Baliraine, F. N., et al. (2011). Quinine, an Old Anti-malarial Drug in a Modern World: Role in the Treatment of Malaria. Malar. J. 10, 144. doi:10.1186/1475-2875-10-144

Adebayo, J. O., and Krettli, A. U. (2011). Potential Antimalarials from Nigerian Plants: A Review. J. Ethnopharmacol. 133, 289-302. doi:10.1016/ j.jep.2010.11.024

AdenAbdi, Y., Gustafsson, L. L., Ericsson, O., and Hellgren, U. (1995). Handbook of Drugs for Tropical Parasitic Infections. 2nd Edn. London, UK: CRC Press.

Aguiar, A. C., Cunha, A. C., Ceravolo, I. P., Gonçalves, R. A., Oliveira, A. J., and Krettli, A. U. (2015). Aspidosperma (Apocynaceae) Plant Cytotoxicity and Activity Towards Malaria Parasites. Part II: Experimental Studies with Aspidosperma Ramiflorum In Vivo and In Vitro. Mem. Inst. Oswaldo Cruz 110, 906-913. doi:10.1590/0074-02760150188

Ahmed, S. M., Nordeng, H., Sundby, J., Aragaw, Y. A., and de Boer, H. J. (2018). The Use of Medicinal Plants by Pregnant Women in Africa: A Systematic Review. J. Ethnopharmacol. 224, 297-313. doi:10.1016/j.jep.2018.05.032

Akuodor, G. C., Essien, D. O., Nkorroh, J. A., Essien, A. D., Nkanor, E. E., Ezeunala, M. N., et al. (2017). Antiplasmodial Activity of the Ethanolic Root Bark Extract of Icacina Senegalensis in Mice Infected by Plasmodium Berghei. J. Basic Clin. Physiol. Pharmacol. 28, 181-184. doi:10.1515/jbcpp-2016-0109

Alebie, G., Urga, B., and Worku, A. (2017). Systematic Review on Traditional Medicinal Plants Used for the Treatment of Malaria in Ethiopia: Trends and Perspectives. Malar. J. 16, 307. doi:10.1186/s12936-017-1953-2

Alli, L. A., Adesokan, A. A., and Salawu, A. O. (2016). Antimalarial Activity of Fractions of Aqueous Extract of Acacia Nilotica Root. J. Intercult. Ethnopharmacol. 5, 180-185. doi:10.5455/jice.20160331064817

Amelo, W., Nagpal, P., and Makonnen, E. (2014). Antiplasmodial Activity of Solvent Fractions of Methanolic Root Extract of Dodonaea Angustifolia in Plasmodium Berghei Infected Mice. BMC Complement. Altern. Med. 14, 462. doi:10.1186/1472-6882-14-462

Andrade-Neto, V. F., Brandão, M. G., Nogueira, F., Rosário, V. E., and Krettli, A. U. (2008). Ampelozyziphus Amazonicus Ducke (Rhamnaceae), a Medicinal Plant Used to Prevent Malaria in the Amazon Region, Hampers the Development of Plasmodium Berghei Sporozoites. Int. J. Parasitol. 38, 1505-1511. doi:10.1016/ j.ijpara.2008.05.007

Andrade-Neto, V. F., Brandão, M. G., Oliveira, F. Q., Casali, V. W., Njaine, B., Zalis, M. G., et al. (2004). Antimalarial Activity of Bidens Pilosa L. (Asteraceae) Ethanol Extracts from Wild Plants Collected in Various Localities or Plants Cultivated in Humus Soil. Phytother. Res. 18, 634-639. doi:10.1002/ptr10.1002/ ptr. 1510

Andrade-Neto, V. F., Brandão, M. G., Stehmann, J. R., Oliveira, L. A., and Krettli, A. U. (2003). Antimalarial Activity of Cinchona-like Plants Used to Treat Fever and Malaria in Brazil. J. Ethnopharmacol. 87, 253-256. doi:10.1016/s03788741(03)00141-7

Ansari, M. T., Saify, Z. S., Sultana, N., Ahmad, I., Saeed-Ul-Hassan, S., Tariq, I., et al. (2013). Malaria and Artemisinin Derivatives: an Updated Review. Mini Rev. Med. Chem. 13, 1879-1902. doi:10.2174/13895575113136660097

Ariey, F., Witkowski, B., Amaratunga, C., Beghain, J., Langlois, A. C., Khim, N., et al. (2014). A Molecular Marker of Artemisinin-Resistant Plasmodium Falciparum Malaria. Nature 505, 50-55. doi:10.1038/nature12876

Asnake, S., Teklehaymanot, T., Hymete, A., Erko, B., and Giday, M. (2015). Evaluation of the Antiplasmodial Properties of Selected Plants in Southern

\section{ACKNOWLEDGMENTS}

All authors are deeply thankful to Dr. Virgilio do Rosario, for his excellent and generous work on the English review. We are also thankful to Professor Ricardo T Gazzinelli for financial support to the fee to publish the review.

Ethiopia. BMC Complement. Altern. Med. 15, 448. doi:10.1186/s12906-0150976-x

Asrade, S., Mengesha, Y., Moges, G., and Gelayee, D. A. (2017). In Vivo antiplasmodial Activity Evaluation of the Leaves of Balanites Rotundifolia (Van Tiegh.) Blatter (Balanitaceae) against Plasmodium Berghei. J. Exp. Pharmacol. 9, 59-66. doi:10.2147/JEP.S130491

Asua, V., Conrad, M. D., Aydemir, O., Duvalsaint, M., Legac, J., Duarte, E., et al. (2021). Changing Prevalence of Potential Mediators of Aminoquinoline, Antifolate, and Artemisinin Resistance across Uganda. J. Infect. Dis. 223, 985-994. doi:10.1093/infdis/jiaa687

Asua, V., Vinden, J., Conrad, M. D., Legac, J., Kigozi, S. P., Kamya, M. R., et al. (2019). Changing Molecular Markers of Antimalarial Drug Sensitivity across Uganda. Antimicrob. Agents Chemother. 63, e01818-18. doi:10.1128/ AAC.01818-18

Attemene, S. D. D., Beourou, S., Tuo, K., Gnondjui, A. A., Konate, A., Toure, A. O., et al. (2018). Antiplasmodial Activity of Two Medicinal Plants against Clinical Isolates of Plasmodium Falciparum and Plasmodium Berghei Infected Mice. J. Parasit. Dis. 42, 68-76. doi:10.1007/s12639-017-0966-7

Azwanida, N. N. (2015). A Review on the Extraction Methods Use in Medicinal Plants, Principle, Strength and Limitation. Med. Aromat. Plants 4, 3. doi:10.4172/2167-0412.1000196

Balint, G. A. (2001). Artemisinin and its Derivatives: an Important New Class of Antimalarial Agents. Pharmacol. Ther. 90, 261-265. doi:10.1016/s01637258(01)00140-1

Bankole, A. E., Adekunle, A. A., Sowemimo, A. A., Umebese, C. E., Abiodun, O., and Gbotosho, G. O. (2016). Phytochemical Screening and In Vivo Antimalarial Activity of Extracts from Three Medicinal Plants Used in Malaria Treatment in Nigeria. Parasitol. Res. 115, 299-305. doi:10.1007/s00436-015-4747-x

Bantie, L., Assefa, S., Teklehaimanot, T., and Engidawork, E. (2014). In Vivo antimalarial Activity of the Crude Leaf Extract and Solvent Fractions of Croton Macrostachyus Hocsht. (Euphorbiaceae) against Plasmodium Berghei in Mice. BMC Complement. Altern. Med. 14, 79-89. doi:10.1186/1472-6882-14-79

Belay, W. Y., Endale Gurmu, A., and Wubneh, Z. B. (2018). Antimalarial Activity of Stem Bark of Periploca Linearifolia during Early and Established Plasmodium Infection in Mice. Evid-based Compl. Alt. Med., 4169397. doi:10.1155/2018/ 4169397

Berthi, W., González, A., Rios, A., Blair, S., Cogollo, Á., and Pabón, A. (2018). Antiplasmodial Effect of Plant Extracts from Picrolemma Huberi and Picramnia Latifolia. Malar. J. 17, 151. doi:10.1186/s12936-018-2301-x

Best Plummer, W., and Pinto Pereira, L. (2008). Diminished Plasmodium Falciparum Sensitivity to Quinine Exposure In Vitro and in a Sequential Multi-Drug Regimen: A Preliminary Investigation in Guyana, South America. Int. J. Infect. Dis. 12, 27-31. doi:10.1016/j.ijid.2008.03.024

Birru, E. M., Geta, M., and Gurmu, A. E. (2017). Antiplasmodial Activity of Indigofera Spicata Root Extract against Plasmodium Berghei Infection in Mice. Malar. J. 16, 198. doi:10.1186/s12936-017-1853-5

Biruk, H., Sentayehu, B., Alebachew, Y., Tamiru, W., Ejigu, A., and Assefa, S. (2020). In Vivo antimalarial Activity of $80 \%$ Methanol and Aqueous Bark Extracts of Terminalia Brownii Fresen. (Combretaceae) against Plasmodium Berghei in Mice. Biochem. Res. Int. 2020, 7. doi:10.1155/ 2020/9749410

Biruksew, A., Zeynudin, A., Alemu, Y., Golassa, L., Yohannes, M., Debella, A., et al. (2018). Zingiber Officinale Roscoe and Echinops Kebericho Mesfin Showed Antiplasmodial Activities against Plasmodium Berghei in a Dose-dependent Manner in Ethiopia. Ethiop. J. Health Sci. 28, 655-664. doi:10.4314/ ejhs.v28i5.17

Bitew, H., Mammo, W., Hymete, A., and Yeshak, M. Y. (2017). Antimalarial Activity of Acetylenic Thiophenes from Echinops Hoehnelii Schweinf. Molecules 22, 1965. doi:10.3390/molecules22111965 
Bjorkman, A., and Phillips-Howard, P. A. (1990). The Epidemiology of Drug-Resistant Malaria. Trans. R. Soc. Trop. Med. Hyg. 84, 177-180. doi:10.1016/0035-9203(90)90246-B

Botelho, M. G. A., Paulino Filho, H. E., and Krettli, A. (1981). Quimioterapia Experimental Antimalárica Usando a Rhamnaceae Ampelozizyphus Amazonicus Ducke, Vulgarmente Denominada Cerveja-De-Índio Contra O Plasmodium Berghei. Oréades 8, 437-442.

Brandão, M. G. L., Botelho, M. G. A., and Krettli, A. (1985). Quimioterapia Experimental Antimalárica Com Produtos Naturais: I. Uma Abordagem Mais Racional? Cien. Cult. 37, 1152-1163.

Brandão, M. G. L., Grandi, T. S. M., Rocha, E. M. M., Sawyer, D. R., and Krettli, A. U. (1992). Survey of Medicinal Plants Used as Antimalarials in the Amazon. J. Ethnopharmacol. 36, 175-182. doi:10.1016/0378-8741(92)90018-M

Buffet, P. A., Safeukui, I., Deplaine, G., Brousse, V., Prendki, V., Thellier, M., et al. (2011). The Pathogenesis of Plasmodium Falciparum Malaria in Humans: Insights from Splenic Physiology. Blood 117, 381-392. doi:10.1182/blood2010-04-202911

Carvalho, L. H., Brandão, M. G., Santos-Filho, D., Lopes, J. L., and Krettli, A. U. (1991). Antimalarial Activity of Crude Extracts from Brazilian Plants Studied In Vivo in Plasmodium Berghei-infected Mice and In Vitro against Plasmodium Falciparum in Culture. Braz. J. Med. Biol. Res. 24, 1113-1123.

Carvalho, L. H., Ferrari, W. M., and Krettli, A. U. (1992). A Method for Screening Drugs against the Liver Stages of Malaria Using Plasmodium Gallinaceum and Aedes Mosquitos. Braz. J. Med. Biol. Res. 25, 247-255.

Ceravolo, I. P., Zani, C. L., Figueiredo, F. J. B., Kohlhoff, M., Santana, A. E. G., and Krettli, A. U. (2018). Aspidosperma Pyrifolium, a Medicinal Plant from the Brazilian Caatinga, Displays a High Antiplasmodial Activity and Low Cytotoxicity. Malar. J. 17, 436. doi:10.1186/s12936018-2568-y

Chandel, S., and Bagai, U. (2011). Screening of Antiplasmodial Efficacy of Ajuga Bracteosa Wall Ex. Benth. Parasitol. Res. 108, 801-805. doi:10.1007/s00436011-2250-6

Chen, S., Wan, L., Couch, L., Lin, H., Li, Y., Dobrovolsky, V. N., et al. (2013). Mechanism Study of Goldenseal-Associated DNA Damage. Toxicol. Lett. 221, 64-72. doi:10.1016/j.toxlet.2013.05.641

Chierrito, T. P., Aguiar, A. C., de Andrade, I. M., Ceravolo, I. P., Gonçalves, R. A. C., de Oliveira, A. J. B., et al. (2014). Anti-malarial Activity of Indole Alkaloids Isolated from Aspidosperma Olivaceum. Malar. J. 13, 142. doi:10.1186/14752875-13-142

China Cooperative Research Group (1982). Chemical Studies on Qinghaosu. Tradit. Chin. Med. 2, 3-8.

Chinsembu, K. C. (2015). Plants as Antimalarial Agents in Sub-saharan Africa. Acta Trop. 152, 32-48. doi:10.1016/j.actatropica.2015.08.009

Christian, A. G., Thecla, E. C., Dick, E. A., Chile, A. E., Chimsorom, C. K., Ckukwu, N. D., et al. (2017). In Vivo antiplasmodial Activity of Bombax Buonopozense Root Bark Aqueous Extract in Mice Infected by Plasmodium Berghei. J. Tradit. Chin. Med. 37, 431-435. doi:10.1016/S0254-6272(17) 30148-6

Chutmongkonkul, M., Maier, W. A., and Seitz, H. M. (1992). A New Model for Testing Gametocytocidal Effects of Some Antimalarial Drugs on Plasmodium Falciparum In Vitro. Ann. Trop. Med. Parasitol. 86, 207-215. doi:10.1080/ 00034983.1992.11812656

Coutinho, J. P., Aguiar, A. C., dos Santos, P. A., Lima, J. C., Rocha, M. G., Zani, C. L., et al. (2013). Aspidosperma (Apocynaceae) Plant Cytotoxicity and Activity towards Malaria Parasites. Part I: Aspidosperma Nitidum (Benth) Used as a Remedy to Treat Fever and Malaria in the Amazon. Mem. Inst. Oswaldo Cruz 108, 974-982. doi:10.1590/0074-0276130246

Crompton, P. D., Moebius, J., Portugal, S., Waisberg, M., Hart, G., Garver, L. S., et al. (2014). Malaria Immunity in Man and Mosquito: Insights into Unsolved Mysteries of a Deadly Infectious Disease. Annu. Rev. Immunol. 32, 157-187. doi:10.1146/annurev-immunol-032713-120220

Cui, L., and Su, X. Z. (2009). Discovery, Mechanisms of Action and Combination Therapy of Artemisinin. Expert Rev. Anti Infect. Ther. 7, 999-1013. doi:10.1586/ eri.09.68

Cysne, D. N., Fortes, T. S., Reis, A. S., de Paulo Ribeiro, B., Dos Santos Ferreira, A., do Amaral, F. M., et al. (2016). Antimalarial Potential of Leaves of Chenopodium Ambrosioides L. Parasitol. Res. 115, 4327-4334. doi:10.1007/ s00436-016-5216-x
De Oliveira, A. R. M., and Szczerbowski, D. (2009). Quinina: 470 anos de história, controvérsias e desenvolvimento. Quim. Nova 32, 1971-1974. doi:10.1590/ S0100-40422009000700048

de Souza, G. A., da Silva, N. C., de Souza, J., de Oliveira, K. R., da Fonseca, A. L., Baratto, L. C., et al. (2017). In Vitro and In Vivo Antimalarial Potential of Oleoresin Obtained from Copaifera Reticulata Ducke (Fabaceae) in the Brazilian Amazon Rainforest. Phytomedicine 24, 111-118. doi:10.1016/ j.phymed.2016.11.021

Derebe, D., and Wubetu, M. (2019). Antimalarial Activity of Hydroalcoholic Root Extract of Acanthus Polystachyus Delile (Acanthaceae) against Plasmodium Berghei-infected Mice. J. Evid. Based Integr. Med. 24, 1-6. doi:10.1177/ 2515690X19885322

Dibessa, T. T., Engidawork, E., Nedi, T., and Teklehaymanot, T. (2020). Antimalarial Activity of the Aqueous Extract of the Latex of Aloe Pirottae Berger. (Aloaceae) against Plasmodium Berghei in Mice. J. Ethnopharmacol. 255, 112763. doi:10.1016/j.jep.2020.112763

Dondorp, A. M., Nosten, F., Yi, P., Das, D., Phyo, A. P., Tarning, J., et al. (2009). Artemisinin Resistance in Plasmodium Falciparum Malaria. N. Engl. J. Med. 361, 455-467. doi:10.1056/NEJMoa0808859

Dondorp, A. M., and Von Seidlein, L. V. (2017). "Malaria" in Infectious Diseases. Editors J. Cohen, W. Powderly, and S. Opal. 1014-1025. doi:10.1016/b978-07020-6285-8.00117-9

Ekasari, W., Putri, A. R. K., Winata, C. A., Putri, N. T., Hamsidi, R., Arwati, H., et al. (2020). Antimalarial Activity of Multiple Dose on Plasmodium Berghei Infected Mice and Heme Detoxification Inhibitory Activity of Helianthus Annuus L. Leaf Extract. J. Pharm. Sci. 45, 145-152.

Ekasari, W., Widya Pratiwi, D., Amanda, Z., Suciati, A., and Arwati, H. (2019). Various Parts of Helianthus Annuus Plants as New Sources of Antimalarial Drugs. Evid-based Compl. Alt. Med. 7390385. doi:10.1155/ 2019/7390385

Enechi, O. C., Amah, C. C., Okagu, I. U., Ononiwu, C. P., Azidiegwu, V. C., Ugwuoke, E. O., et al. (2019). Methanol Extracts of Fagara Zanthoxyloides Leaves Possess Antimalarial Effects and Normalizes Haematological and Biochemical Status of Plasmodium Berghei-passaged Mice. Pharm. Biol. 57, 577-585. doi:10.1080/13880209.2019.1656753

Falade, M. O., Akinboye, D. O., Gbotosho, G. O., Ajaiyeoba, E. O., Happi, T. C., Abiodun, O. O., et al. (2014). In Vitro and In Vivo Antimalarial Activity of Ficus Thonningii Blume (Moraceae) and Lophira Alata Banks (Ochnaceae), Identified from the Ethnomedicine of the Nigerian Middle belt. J. Parasitol. Res. 972853. doi:10.1155/2014/972853

Fenta, M., and Kahaliw, W. (2019). Evaluation of Antimalarial Activity of Hydromethanolic Crude Extract and Solvent Fractions of the Leaves of Nuxia Congesta R. Br. Ex Fresen (Buddlejaceae) in Plasmodium Berghei Infected Mice. J. Exp. Pharmacol. 11, 121-134. doi:10.2147/JEP.S230636

Fentahun, S., Makonnen, E., Awas, T., and Giday, M. (2017). In Vivo antimalarial Activity of Crude Extracts and Solvent Fractions of Leaves of Strychnos Mitis in Plasmodium Berghei Infected Mice. BMC Complementaltern. Med. 17, 13. doi:10.1186/s12906-016-1529-7

Ferreira, L. T., Venancio, V. P., Kawano, T., Abrão, L. C. C., Tavella, T. A., Almeida, L. D., et al. (2019). Chemical Genomic Profiling Unveils the In Vitro and In Vivo Antiplasmodial Mechanism of Açaí (Euterpe Oleracea Mart.) Polyphenols. ACS Omega 4, 15628-15635. doi:10.1021/acsomega.9b02127

Fidock, D. A., Rosenthal, P. J., Croft, S. L., Brun, R., and Nwaka, S. (2004). Antimalarial Drug Discovery: Efficacy Models for Compound Screening. Nat. Rev. Drug Discov. 3, 509-520. doi:10.1038/nrd1416

Gachelin, G., Garner, P., Ferroni, E., Tröhler, U., and Chalmers, I. (2017). Evaluating Cinchona Bark and Quinine for Treating and Preventing Malaria. J. R. Soc. Med. 110, 31-40. doi:10.1177/014107681668142110.1177/ 0141076816688411

Girma, S., Giday, M., Erko, B., and Mamo, H. (2015). Effect of Crude Leaf Extract of Osyris Quadripartita on Plasmodium Berghei in Swiss Albino Mice. BMC Complement. Altern. Med. 15, 1-9. doi:10.1186/s12906-0150715-3

Greenwood, B. M., Fidock, D. A., Kyle, D. E., Kappe, S. H. I., Alonso, P. L., Collins, F. H., et al. (2008). Malaria: Progress, Perils, and Prospects for Eradication. J. Clin. Invest. 118, 1266-1276. doi:10.1172/JCI33996

Guerra, F. (1977). The Introduction of Cinchona in the Treatment of Malaria. Part I. J. Trop. Med. Hyg. 80, 112-118. 
Habtemariam, S., and Dagne, E. (2009). Prooxidant Action of Knipholone Anthrone: Copper Dependent Reactive Oxygen Species Generation and DNA Damage. Food Chem. Toxicol. 47, 1490-1494. doi:10.1016/ j.fct.2009.03.032

Haddad, M., Mahbodfar, H., Zamani, Z., and Ramazani, A. (2017). Antimalarial Evaluation of Selected Medicinal Plant Extracts Used in Iranian Traditional Medicine. Iran. J. Basic Med. Sci. 20, 415-422. doi:10.22038/ IJBMS.2017.8583

Haidara, M., Haddad, M., Denou, A., Marti, G., Bourgeade-Delmas, S., Sanogo, R., et al. (2018). In Vivo validation of Anti-malarial Activity of Crude Extracts of Terminalia Macroptera, a Malian Medicinal Plant. Malar. J. 17, 68. doi:10.1186/ s12936-018-2223-7

Hailesilase, G. G., Rajeshwar, Y., Hailu, G. S., Sibhat, G. G., and Bitew, H. (2020). In Vivo antimalarial Evaluation of Crude Extract, Solvent Fractions, and TLCIsolated Compounds from Olea Europaea Linn Subsp. Cuspidata (Oleaceae). Evid-based Compl. Alt. Med. 2020, 6731485. doi:10.1155/2020/6731485

Hien, T. T., and White, N. J. (1993). Qinghaosu. Lancet 341, 603-608. doi:10.1016/ 0140-6736(93)90362-k

Howard, B. F. (1931). Some Notes on the Cinchona Industry. Chem. News 142, 129-133. doi:10.1126/science.73.1883.129

Hsu, E. (2006). The History of Qing Hao in the Chinese Materia Medica. Trans. $R$. Soc. Trop. Med. Hyg. 100, 505-508. doi:10.1016/j.trstmh.2005.09.020

Induli, M., Gebru, M., Abdissa, N., Akala, H., Wekesa, I., Byamukama, R., et al. (2013). Antiplasmodial Quinones from the Rhizomes of Kniphofia Foliosa. Nat. Prod. Commun. 8, 1261-1264. doi:10.1177/1934578X1300800920

Institute of Medicine (2004). Saving Lives, Buying Time: Economics of Malaria Drugs in an Age of Resistance. Washington, DC: The National Academies Press, 126-128.

Jansen, O., Tits, M., Angenot, L., Nicolas, J. P., De Mol, P., Nikiema, J. B., et al. (2012). Anti-plasmodial Activity of Dicoma Tomentosa (Asteraceae) and Identification of Urospermal A-15-O-Acetate as the Main Active Compound. Malar. J. 11, 289. doi:10.1186/1475-2875-11-289

Jaramillo-Arango, J. (1949). A Critical Review of the Basic Facts in the History of Cinchona. Biol. J. Linn. Soc. Lond. 53, 272-311. doi:10.1111/j.10958339.1949.tb00419.x

Jarcho, S., and Torti, F. (1993). Quinine's Predecessor: Francesco Torti and the Early History of Cinchona. Baltimore, MD: John Hopkins University Press, 154-155.

Jelodar, N. B., Bhatt, A., and Mohamed, K. (2014). New Cultivation Approaches of Artemisia Annua L. For a Sustainable Production of the Antimalarial Drug Artemisinin. J. Med. Plant Res. 8, 441-447. doi:10.5897/JMPR11.1053

Jennings, B. R., and Ridler, P. J. (1983). Interaction of Chromosomal Stains with DNA. An Electrofluorescence Study. Biophys. Struct. Mech. 10, 71-79. doi:10.1007/BF00535543

Johnson, T. O., Istifanus, G., and Kutshik, R. J. (2020). In Vitro and In Vivo Analysis of the Anti-plasmodial Activity of Ethanol Extract of Phyllanthus Nivosus W. Bull. Leaf. J. Parasit. Dis. 44, 166-173. doi:10.1007/s12639-01901178-4

Kalani, K., Agarwal, J., Alam, S., Khan, F., Pal, A., and Srivastava, S. K. (2013). In Silico and In Vivo Anti-malarial Studies of $18 \beta$ Glycyrrhetinic Acid from Glycyrrhiza Glabra. PLOS One 8, e74761. doi:10.1371/journal.pone.0074761

Kamaraj, C., Rahuman, A. A., Roopan, S. M., Bagavan, A., Elango, G., Zahir, A. A., et al. (2014). Bioassay-guided Isolation and Characterization of Active Antiplasmodial Compounds from Murraya Koenigii Extracts against Plasmodium Falciparum and Plasmodium Berghei. Parasitol. Res. 113, 1657-1672. doi:10.1007/s00436-014-3810-3

Kamkumo, R. G., Ngoutane, A. M., Tchokouaha, L. R., Fokou, P. V., Madiesse, E. A., Legac, J., et al. (2012). Compounds from Sorindeia Juglandifolia (Anacardiaceae) Exhibit Potent Anti-plasmodial Activities In Vitro and In Vivo. Malar. J. 11, 382. doi:10.1186/1475-2875-11-382

Kaufman, T. S., and Rúveda, E. A. (2005). The Quest for Quinine: Those Who Won the Battles and Those Who Won the War. Angew. Chem. Int. Ed. Engl. 44, 854-885. doi:10.1002/anie.200400663

Kayano, A. C., Lopes, S. C., Bueno, F. G., Cabral, E. C., Souza-Neiras, W. C., Yamauchi, L. M., et al. (2011). In Vitro and In Vivo Assessment of the Antimalarial Activity of Caesalpinia Pluviosa. Malar. J. 10, 112. doi:10.1186/14752875-10-112

Kefe, A., Giday, M., Mamo, H., and Erko, B. (2016). Antimalarial Properties of Crude Extracts of Seeds of Brucea Antidysenterica and Leaves of Ocimum
Lamiifolium. BMC Complement. Altern. Med. 16, 118. doi:10.1186/s12906-0161098-9

Kheir, M. M., Wang, Y., Hua, L., Hu, J., Li, L., Lei, F., et al. (2010). Acute Toxicity of Berberine and its Correlation with the Blood Concentration in Mice. Food Chem. Toxicol. 48, 1105-1110. doi:10.1016/j.fct.2010.01.033

Kifle, Z. D., Adinew, G. M., Mengistie, M. G., Gurmu, A. E., Enyew, E. F., Goshu, B. T., et al. (2020). Evaluation of Antimalarial Activity of Methanolic Root Extract of Myrica Salicifolia A Rich (Myricaceae) Against Plasmodium Berghei-Infected Mice. J. Evid. Based Integr. Med. 25, 2515690X20920539. doi:10.1177/ 2515690X20920539

Kifle, Z. D., and Atnafie, S. A. (2020). Anti-oxidant Potential and Antimalarial Effects of Acanthus Polystachyus Delile (Acanthaceae) against Plasmodium Berghei: Evidence for In Vivo Antimalarial Activity. J. Exp. Pharmacol. 12, 575-587. doi:10.2147/JEP.S282407

Kiraithe, M. N., Nguta, J. M., Mbaria, J. M., and Kiama, S. G. (2016). Evaluation of the Use of Ocimum Suave Willd. (Lamiaceae), Plectranthus Barbatus Andrews (Lamiaceae) and Zanthoxylum Chalybeum Engl. (Rutaceae) as Antimalarial Remedies in Kenyan Folk Medicine. J. Ethnopharmacol. 178, 266-271. doi:10.1016/j.jep.2015.12.013

Klayman, D. L. (1985). Qinghaosu (Artemisinin): an Antimalarial Drug from China. Science 228, 1049-1055. doi:10.1126/science.3887571

Krettli, A. U., Andrade-Neto, V. F., Brandão, M. G., and Ferrari, W. M. (2001). The Search for New Antimalarial Drugs from Plants Used to Treat Fever and Malaria or Plants Ramdomly Selected: a Review. Mem. Inst. Oswaldo Cruz 96, 1033-1042. doi:10.1590/S0074-02762001000800002

Krishna, S., Bustamante, L., Haynes, R. K., and Staines, H. M. (2008). Artemisinins: Their Growing Importance in Medicine. Trends Pharmacol. Sci. 29, 520-527. doi:10.1016/j.tips.2008.07.004

Kutchan, T. M. (2001). Ecological Arsenal and Developmental Dispatcher. The Paradigm of Secondary Metabolism. Plant Physiol. 125, 58-60. doi:10.1104/ pp.125.1.58

Kweyamba, P. A., Zofou, D., Efange, N., Assob, J. C. N., Kitau, J., and Nyindo, M. (2019). In Vitro and In Vivo Studies on Anti-malarial Activity of Commiphora Africana and Dichrostachys Cinerea Used by the Maasai in Arusha Region, Tanzania. Malar. J. 18, 119. doi:10.1186/s12936-019-2752-8

Lahlou, M. (2007). Screening of Natural Products for Drug Discovery. Expert Opin. Drug Discov. 2, 697-705. doi:10.1517/17460441.2.5.697

Lampah, D. A., Yeo, T. W., Hardianto, S. O., Tjitra, E., Kenangalem, E., Sugiarto, P., et al. (2011). Coma Associated with Microscopy-Diagnosed Plasmodium Vivax: A Prospective Study in Papua, Indonesia. Plos Negl. Trop. Dis. 5, 1032. doi:10.1371/journal.pntd.0001032

Lang, L., Hu, Q., Wang, J., Liu, Z., Huang, J., Lu, W., et al. (2018). Coptisine, a Natural Alkaloid from Coptidis Rhizoma, Inhibits Plasmodium Falciparum Dihydroorotate Dehydrogenase. Chem. Biol. Drug Des. 92, 1324-1332. doi:10.1111/cbdd.13197

Lee, M. R. (2002). Plants against Malaria. Part 1: Cinchona or the Peruvian Bark. J. R. Coll. Physicians Edinb. 32, 189-196. doi:10.1007/978-3-66204897-9_19

Legrand, E., Volney, B., Meynard, J. B., Mercereau-Puijalon, O., and Esterre, P. (2008). Vitro Monitoring of Plasmodium Falciparum Drug Resistance in French Guiana: a Synopsis of Continuous Assessment from 1994 to 2005. Antimicrob. Agents Chemother. 52, 288-298. doi:10.1128/ AAC.00263-07

Li, Y., Luan, Y., Qi, X., Li, M., Gong, L., Xue, X., et al. (2010). Emodin Triggers DNA Double-Strand Breaks by Stabilizing Topoisomerase II-DNA Cleavage Complexes and by Inhibiting ATP Hydrolysis of Topoisomerase II. Toxicol. Sci. 118, 435-443. doi:10.1093/toxsci/kfq282

Lima, R. B., Rocha e Silva, L. F., Melo, M. R., Costa, J. S., Picanço, N. S., Lima, E. S., et al. (2015). In Vitro and In Vivo Anti-malarial Activity of Plants from the Brazilian Amazon. Malar. J. 14, 508. doi:10.1186/s12936-015-0999-2

Liu, J. M., Ni, M. Y., Fan, J. F., Tu, Y. Y., Wu, Z. H., Wu, Y. L., et al. (1979). Structure and Reaction of Arteannuin. Acta Chim. Sin. 37, 129-143.

Lorke, D. (1983). A New Approach to Practical Acute Toxicity Testing. Arch. Toxicol. 54, 275-287. doi:10.1007/BF01234480

Maranz, S. (2012). An Alternative Paradigm for the Role of Antimalarial Plants in Africa. Sci. World J. 978913. doi:10.1100/2012/978913

Mathieu, L. C., Cox, H., Early, A. M., Mok, S., Lazrek, Y., Paquet, J. C., et al. (2020). Local Emergence in Amazonia of Plasmodium Falciparum K13 C580Y Mutants 
Associated with In Vitro Artemisinin Resistance. Elife 9, e51015. doi:10.7554/ eLife.51015

Maude, R. J., Woodrow, C. J., and White, L. J. (2010). Artemisinin Antimalarials: Preserving the "magic Bullet. Drug Dev. Res. 71, 12-19. doi: $10.1002 / \mathrm{ddr} .20344$

Mayxay, M., Barends, M., Brockman, A., Jaidee, A., Nair, S., Sudimack, D., et al. (2007). In Vitro antimalarial Drug Susceptibility and Pfcrt Mutation Among Fresh Plasmodium Falciparum Isolates from the Lao PDR (Laos). Am. J. Trop. Med. Hyg. 76, 245-250. doi:10.4269/ajtmh.2007.76.245

Memvanga, P. B., Tona, G. L., Mesia, G. K., Lusakibanza, M. M., and Cimanga, R. K. (2015). Antimalarial Activity of Medicinal Plants from the Democratic Republic of Congo: A Review. J. Ethnopharmacol. 169, 76-98. doi:10.1016/ j.jep.2015.03.075

Mendanha da Cunha, C. R., Mendanha Neto, S. A., Carlos da Silva, C., Cortez, A. P., Gomes, M. N., Martins, F. I., et al. (2013). 4-Nerolidylcatechol and its Synthetic Analogues: Antioxidant Activity and Toxicity Evaluation. Eur. J. Med. Chem. 62, 371-378. doi:10.1016/j.ejmech.2012.12.028

Menkin-Smith, L., and Winders, W. T. (2020). Plasmodium Vivax Malaria. Treasure Island, FL: StatPearls.

Mesfin, A., Giday, M., Animut, A., and Teklehaymanot, T. (2012). Ethnobotanical Study of Antimalarial Plants in Shinile District, Somali Region, Ethiopia, and In Vivo Evaluation of Selected Ones against Plasmodium Berghei. J. Ethnopharmacol. 139, 221-227. doi:10.1016/j.jep.2011.11.006

Meshnick, S. R. (1998). Artemisinin Antimalarials: Mechanism of Action and Resistance. Med. Trop. (Mars) 58, 13-17.

Mi, R., Tu, B., Bai, X. T., Chen, J., Ouyang, Y., and Hu, Y. J. (2015). Binding Properties of Palmatine to DNA: Spectroscopic and Molecular Modeling Investigations. Luminescence 30, 1344-1351. doi:10.1002/bio.2904

Ministério, d. S. (2020). Malária. Boletim Epidemiológico Secretaria de Vigilância em Saúde. Available at: https://www.gov.br/saude/pt-br/assuntos/media/pdf/ 2020/dezembro/03/boletim_especial_malaria_1dez20_final.pdf (Accessed June 21, 2021).

Miotto, O., Sekihara, M., Tachibana, S. I., Yamauchi, M., Pearson, R. D., Amato, R., et al. (2020). Emergence of Artemisinin-Resistant Plasmodium Falciparum with Kelch13 C580Y Mutations on the Island of New Guinea. Plos Pathog. 16, e1009133. doi:10.1371/journal.ppat.1009133

Misganaw, D., Amare, G. G., and Mengistu, G. (2020). Chemo Suppressive and Curative Potential of Hypoestes Forskalei against Plasmodium Berghei: Evidence for In Vivo Antimalarial Activity. J. Exp. Pharmacol. 12, 313-323. doi:10.2147/ JEP.S262026

Misganaw, D., Engidawork, E., and Nedi, T. (2019). Evaluation of the Anti-malarial Activity of Crude Extract and Solvent Fractions of the Leaves of Olea Europaea (Oleaceae) in Mice. BMC Complement. Altern. Med. 19, 171. doi:10.1186/ s12906-019-2567-8

Mohammed, T., Erko, B., and Giday, M. (2014). Evaluation of Antimalarial Activity of Leaves of Acokanthera Schimperi and Croton Macrostachyus against Plasmodium Berghei in Swiss Albino Mice. BMC Complement. Altern. Med. 14, 314. doi:10.1186/1472-6882-14-314

Mota, M. L., Lobo, L. T., Costa, J. M., Costa, L. S., Rocha, H. A., Rocha e Silva, L. F., et al. (2012). In Vitro and In Vivo Antimalarial Activity of Essential Oils and Chemical Components from Three Medicinal Plants Found in Northeastern Brazil. Planta Med. 78, 658-664. doi:10.1055/s-0031-1298333

Moyo, M., Aremu, A. O., and Van Staden, J. (2015). Medicinal Plants: An Invaluable, Dwindling Resource in Sub-saharan Africa. J. Ethnopharmacol. 174, 595-606. doi:10.1016/j.jep.2015.04.034

Musila, M. F., Dossaji, S. F., Nguta, J. M., Lukhoba, C. W., and Munyao, J. M. (2013). In Vivo antimalarial Activity, Toxicity and Phytochemical Screening of Selected Antimalarial Plants. J. Ethnopharmacol. 146, 557-561. doi:10.1016/ j.jep.2013.01.023

Mutanda, L. N. (1999). Assessment of Drug Resistance to the Malaria Parasite in Residents of Kampala, Uganda. East. Afr. Med. J. 76, 421-424.

Mwangi, G. G., Wagacha, J. M., Nguta, J. M., and Mbaria, J. M. (2015). Brine Shrimp Cytotoxicity and Antimalarial Activity of Plants Traditionally Used in Treatment of Malaria in Msambweni District. Pharm. Biol. 53, 588-593. doi:10.3109/13880209.2014.935861

Mzena, T., Swai, H., and Chacha, M. (2018). Antimalarial Activity of Cucumis Metuliferus and Lippia Kituiensis against Plasmodium Berghei Infection in Mice. Res. Rep. Trop. Med. 9, 81-88. doi:10.2147/RRTM.S150091
Nadia, N. A. C., Cédric, Y., Christian, N. O., Raoul, S. N. S., Jeanette, Y., Abdel Azizi, M., et al. (2020). In Vivo antiplasmodial Activity of Entandrophragma Cylindricum (Sprague) sprague Ethyl Acetate Extract in Plasmodium Bergheiinfected Mice. J. Parasitol. Res. 2020, 8846067. doi:10.1155/2020/8846067

Neiva, A. (1987). Memória das memórias [Memoir of the Memorias. Development of malaria hematozoa resistant to quinine. By Arthur Neiva, 1910]. Mem. Inst. Oswaldo Cruz 82, 303-309.

Nguta, J. M., and Mbaria, J. M. (2013). Brine Shrimp Toxicity and Antimalarial Activity of Some Plants Traditionally Used in Treatment of Malaria in Msambweni District of Kenya. J. Ethnopharmacol. 148, 988-992. doi:10.1016/j.jep.2013.05.053

Nieves, K., Prudhomme, J., Le Roch, K. G., Franzblau, S. G., and Rodríguez, A. D. (2016). Natural Product-Based Synthesis of Novel Antiinfective Isothiocyanateand Isoselenocyanate-Functionalized Amphilectane Diterpenes. Bioorg. Med. Chem. Lett. 26, 854-857. doi:10.1016/j.bmcl.2015.12.080

Noedl, H., Se, Y., Schaecher, K., Smith, B. L., Socheat, D., and Fukuda, M. M. (2008). Evidence of Artemisinin-Resistant Malaria in Western Cambodia. $N$. Eng. J. Med. 359, 2619-2620. doi:10.1056/NEJMc0805011

Nondo, R. S., Erasto, P., Moshi, M. J., Zacharia, A., Masimba, P. J., and Kidukuli, A. W. (2016). In Vivo antimalarial Activity of Extracts of Tanzanian Medicinal Plants Used for the Treatment of Malaria. J. Adv. Pharm. Technol. Res. 7, 59-63. doi:10.4103/2231-4040.179748

Nozaka, T., Watanabe, F., Tadaki, S., Ishino, M., Morimoto, I., Kunitomo, J., et al. (1990). Mutagenicity of Isoquinoline Alkaloids, Especially of the Aporphine Type. Mutat. Res. 240, 267-279. doi:10.1016/0165-1218(90)90077-f

Nureye, D., Assefa, S., Nedi, T., and Engidawork, E. (2018). Vivo Antimalarial Activity of the $80 \%$ Methanolic Root Bark Extract and Solvent Fractions of Gardenia Ternifolia Schumach. \& Thonn. (Rubiaceae) against Plasmodium Berghei. Evid-based Compl. Alt. Med., 9217835. doi:10.1155/2018/9217835

Oguntibeju, O. O. (2018). Medicinal Plants with Anti-inflammatory Activities from Selected Countries and Regions of Africa. J. Inflamm. Res. 11, 307-317. doi:10.2147/JIR.S167789

Oliveira, D. R., Costa, A. L., Leitão, G. G., Castro, N. G., Santos, J. P., and Leitão, S. G. (2011). Ethnopharmacology Study of Saracuramirá (Ampelozizyphus Amazonicus Ducke) in the "Quilombola" Communities of Oriximiná, Pará State, Brazil. Acta Amazon 41, 383-392. doi:10.1590/s004459672011000300008

Oliveira, D. R., Krettli, A. U., Aguiar, A. C., Leitão, G. G., Vieira, M. N., Martins, K. S., et al. (2015). Ethnopharmacological Evaluation of Medicinal Plants Used against Malaria by Quilombola Communities from Oriximiná, Brazil. J. Ethnopharmacol. 173, 424-434. doi:10.1016/j.jep.2015.07.035

Oliveira, F. Q., Andrade-Neto, V., Krettli, A. U., and Brandão, M. G. (2004). New Evidences of Antimalarial Activity of Bidens Pilosa Roots Extract Correlated with Polyacetylene and Flavonoids. J. Ethnopharmacol. 93, 39-42. doi:10.1016/ j.jep.2004.03.026

Omara, T., Kiprop, A. K., Ramkat, R. C., Cherutoi, J., Kagoya, S., Moraa Nyangena, D., et al. (2020). Medicinal Plants Used in Traditional Management of Cancer in Uganda: A Review of Ethnobotanical Surveys, Phytochemistry, and Anticancer Studies. Evid-based Compl. Alt. Med. 3529081. doi:10.1155/2020/3529081

Oro, L., and Wretlind, A. (1961). Pharmacological Effects of Fatty Acids, Triolein and Cottonseed Oil. Acta Pharmacol. Toxicol. 18, 141-152. doi:10.1111/j.16000773.1961.tb00323.x

Ouji, M., Augereau, J. M., Paloque, L., and Benoit-Vical, F. (2018). Plasmodium Falciparum Resistance to Artemisinin-Based Combination Therapies: A Sword of Damocles in the Path toward Malaria Elimination. Parasite 25, 24. doi:10.1051/parasite/2018021

Palhares, R. M., Drummond, M. G., Dos Santos Alves Figueiredo Brasil, B., Cosenza, G. P., Brandão, M. G. L., and Oliveira, G. (2015). Medicinal Plants Recommended by the World Health Organization: DNA Barcode Identification Associated with Chemical Analyses Guarantees Their Quality. PLOS One 10, e0127866. doi:10.1371/journal.pone.0127866

Pan American Health Organization (2017). Report on the Situation of Malaria in the Americas - 2017. Available at: https://www.paho.org/hq/index.php? option $=$ com_topics\&view=article\&id=33\&Itemid $=40757 \&$ lang $=$ en $\quad$ (Accessed June 29, 2021).

Pasqual, M. S., Lauer, C. P., Moyna, P., and Henriques, J. A. (1993). Genotoxicity of the Isoquinoline Alkaloid Berberine in Prokaryotic and 
Eukaryotic Organisms. Mutat. Res. 286, 243-252. doi:10.1016/00275107(93)90189-m

Patwardhan, B., Vaidya, A., and Chorghade, M. (2004). Ayurveda and Natural Products Drug Discovery. Curr. Sci. 86, 789-799.

Pelletier, P. J., and Caventou, J. B. (1820). Recherches Chimiques sur les Quinquinas. Ann. Chim. Phys. 15, 289-318.

Peters, W. (1982). Antimalarial Drug Resistance: an Increasing Problem. Br. Med. Bull. 38, 187-192. doi:10.1093/oxfordjournals.bmb.a071757

Peters, W. (1965). Drug Resistance in Plasmodium Berghei Vincke and Lips, 1948. I. Chloroquine Resistance. Exp. Parasitol. 17, 80-89. doi:10.1016/00144894(65)90012-3

Pillay, P., Maharaj, V. J., and Smith, P. J. (2008). Investigating South African Plants as a Source of New Antimalarial Drugs. J. Ethnopharmacol. 119, 438-454. doi:10.1016/j.jep.2008.07.003

Pradines, B., Mabika Mamfoumbi, M., Parzy, D., Owono Medang, M., Lebeau, C., Mourou Mbina, J. R., et al. (1998). In Vitro susceptibility of Gabonese Wild Isolates of Plasmodium Falciparum to Artemether, and Comparison with Chloroquine, Quinine, Halofantrine and Amodiaquine. Parasitology 6, 541-545. doi:10.1017/s0031182098003400

Price, R. N., Nosten, F., Luxemburger, C., Kuile, F. O., Paiphun, L., Price, R. N., et al. (1996). Effects of Artemisinin Derivatives on Malaria Transmissibility. Lancet 347, 1654-1655. doi:10.1016/S0140-6736(96)91488-9

Qinghaosu Antimalaria Coordinating Research Group (1979). Antimalarial Studies on Qinghaosu. Chin. Med. J. 92, 811-816.

Rajendran, C., Begam, M., Kumar, D., Baruah, I., Gogoi, H. K., Srivastava, R. B., et al. (2014). Antiplasmodial Activity of Certain Medicinal Plants against Chloroquine Resistant Plasmodium Berghei Infected white Albino BALB/c Mice. J. Parasit. Dis. 38, 148-152. doi:10.1007/s12639013-0252-2

Rasoanaivo, P., Ramanitrahasimbola, D., Rafatro, H., Rakotondramanana, D., Robijaona, B., Rakotozafy, A., et al. (2004). Screening Extracts of Madagascan Plants in Search of Antiplasmodial Compounds. Phytother. Res. 18, 742-747. doi:10.1002/ptr.1533

Rocha e Silva, L. F., Nogueira, K. L., Pinto, A. C., Katzin, A. M., Sussmann, R. A., Muniz, M. P., et al. (2015). In Vivo antimalarial Activity and Mechanisms of Action of 4-nerolidylcatechol Derivatives. Antimicrob. Agents Chemother. 59, 3271-3280. doi:10.1128/AAC.05012-14

Rocha e Silva, L. F., Silva Pinto, A. C., Pohlit, A. M., Quignard, E. L., Vieira, P. P., Tadei, W. P., et al. (2011). In Vivo and In Vitro Antimalarial Activity of 4nerolidylcatechol. Phytother. Res. 25, 1181-1188. doi:10.1002/ptr.3424

Roussel, C., Caumes, E., Thellier, M., Ndour, P. A., Buffet, P. A., and Jauréguiberry, S. (2017). Artesunate to Treat Severe Malaria in Travellers: Review of Efficacy and Safety and Practical Implications. J. Trav. Med 24. doi:10.1093/jtm/taw093

Ruiz-Irastorza, G., and Khamashta, M. A. (2008). Hydroxychloroquine: the Cornerstone of Lupus Therapy. Lupus 17, 271-273. doi:10.1177/ 0961203307086643

Sachdeva, C., Mohanakrishnan, D., Kumar, S., and Kaushik, N. K. (2020). Assessment of In Vitro and In Vivo Antimalarial Efficacy and GCFingerprints of Selected Medicinal Plant Extracts. Exp. Parasitol. 219, 108011. doi:10.1016/j.exppara.2020.108011

Sajjadi, S. E., Pestechian, N., Kazemi, M., Mohaghegh, M. A., and Hosseini-Safa, A. (2016). Evaluation of the Antimalarial Effect of Ferulago Angulata (Schlecht.) Boiss. Extract and Suberosin Epoxide against Plasmodium Berghei in Comparison with Chloroquine Using In-Vivo Test. Iran J. Pharm. Res. 15, 515-521.

Satish, P. V. V., Kumari, D. S., and Sunita, K. (2017). Antiplasmodial Efficacy of Calotropis Gigantea (L.) against Plasmodium Falciparum (3D7 Strain) and Plasmodium Berghei (ANKA). J. Vector Borne Dis. 54, 215-225. doi:10.4103/ 0972-9062.217612

Satish, P. V. V., and Sunita, K. (2017). Antimalarial Efficacy of Pongamia Pinnata (L) Pierre against Plasmodium Falciparum (3D7 Strain) and Plasmodium Berghei (ANKA). BMC Complement. Altern. Med. 17, 458. doi:10.1186/ s12906-017-1958-y

Singh, M., Suryanshu, K., Singh, G., Dubey, A., and Chaitanya, R. K. (2021). Plasmodium's Journey through the Anopheles Mosquito: A Comprehensive Review. Biochimie 181, 176-190. doi:10.1016/j.biochi.2020.12.009
Sirignano, C., Snene, A., Tenoh, A. R., El Mokni, R., Rigano, D., Habluetzel, A., et al. (2019). Daucovirgolides I-L, Four Congeners of the Antimalarial Daucovirgolide G from Daucus Virgatus. Fitoterapia 137, 104188. doi:10.1016/j.fitote.2019.104188

Slater, H. C., Griffin, J. T., Ghani, A. C., and Okell, L. C. (2016). Assessing the Potential Impact of Artemisinin and Partner Drug Resistance in Sub-saharan Africa. Malar. J. 15, 10. doi:10.1186/s12936-015-1075-7

Smith, A. C., and Williams, R. M. (2008). Rabe Rest in Peace: Confirmation of the Rabe-Kindler Conversion Ofd-Quinotoxine into Quinine: Experimental Affirmation of the woodward-doering Formal Total Synthesis of Quinine. Angew. Chem. Int. Ed. Engl. 47, 1736-1740. doi:10.1002/anie.200705421

Soh, P. N., and Benoit-Vical, F. (2007). Are West African Plants a Source of Future Antimalarial Drugs? J. Ethnopharmacol. 114, 130-140. doi:10.1016/ j.jep.2007.08.012

Somsak, V., Borkaew, P., Klubsri, C., Dondee, K., Bootprom, P., and Saiphet, B. (2016). Antimalarial Properties of Aqueous Crude Extracts of Gynostemma Pentaphyllum and Moringa Oleifera Leaves in Combination with Artesunate in Plasmodium Berghei-infected Mice. J. Trop. Med. 2016, 8031392. doi:10.1155/ 2016/8031392

Sriram, D., Rao, V. S., Chandrasekhara, K. V., and Yogeeswari, P. (2004). Progress in the Research of Artemisinin and its Analogues as Antimalarials: an Update. Nat. Prod. Res. 18, 503-527. doi:10.1080/14786410310001620556

Sriwilaijareon, N., Petmitr, S., Mutirangura, A., Ponglikitmongkol, M., and Wilairat, P. (2002). Stage Specificity of Plasmodium Falciparum Telomerase and its Inhibition by Berberine. Parasitol. Int. 51, 99-103. doi:10.1016/s13835769(01)00092-7

Sucharit, P., Suntharasamai, P., Chintana, T., and Harinasuta, T. (1979). In Vivo and In Vitro Studies of Quinine Sensitivity of Plasmodium Falciparum in Thailand. Southeast. Asian J. Trop. Med. Public Health 10, 138-141.

Tanaka, T. Q., and Williamson, K. C. (2011). A Malaria Gametocytocidal Assay Using Oxidoreduction Indicator, alamarBlue. Mol. Biochem. Parasitol. 177, 160-163. doi:10.1016/j.molbiopara.2011.02.005

Tanwar, G. S., Khatri, P. C., Sengar, G. S., Kochar, A., Kochar, S. K., Middha, S., et al. (2011). Clinical Profiles of 13 Children with Plasmodium Vivax Cerebral Malaria. Ann. Trop. Paediatr. 31, 351-356. doi:10.1179/ 1465328111Y.0000000040

Teka, T., Awgichew, T., and Kassahun, H. (2020). Antimalarial Activity of the Leaf Latex of Aloe Weloensis (Aloaceae) against Plasmodium Berghei in Mice. J. Trop. Med. 2020, 1397043. doi:10.1155/2020/1397043

Teklemichael, A. A., Mizukami, S., Toume, K., Mosaddeque, F., Kamel, M. G., Kaneko, O., et al. (2020). Anti-malarial Activity of Traditional Kampo Medicine Coptis Rhizome Extract and its Major Active Compounds. Malar. J. 19, 204. doi:10.1186/s12936-020-03273-x

Teklu, T., Engidawork, E., Nedi, T., Teklehaymanot, T., and Gebremeskel, L. (2020). Evaluation of the Antimalarial Activity of the Hydroalcoholic Extract of Leaf of Leonotis Ocymifolia (Burm. f.) Iwarsson (Lamiaceae) against Plasmodium Berghei in Mice. Evid-based Compl. Alt. Med. 2020, 5384804. doi:10.1155/2020/5384804

Toma, A., Deyno, S., Fikru, A., Eyado, A., and Beale, A. (2015). In Vivo antiplasmodial and Toxicological Effect of Crude Ethanol Extract of Echinops Kebericho Traditionally Used in Treatment of Malaria in Ethiopia. Malar. J. 14, 196. doi:10.1186/s12936-015-0716-1

Tometsko, A. M., Torous, D. K., and Dertinger, S. D. (1993). Analysis of Micronucleated Cells by Flow Cytometry. 1. Achieving High Resolution with a Malaria Model. Mutat. Res. 292, 129-135. doi:10.1016/0165-1161(93) 90140-u

Tshisekedi, T. P., Mutwale, K. P., Kabongo, K. M. J., Tujibikila, M. A., Kalenda, D. T., Tchinda, A. T., et al. (2017). Antiplasmodial Activity of Heinsia Crinita (Rubiaceae) and Identification of New Iridoids. J. Ethnopharmacol. 196, 261-266. doi:10.1016/j.jep.2016.11.041

Turner, R. B., and Woodward, R. B. (1998). "The Chemistry of the Cinchona Alkaloids," in The Alkaloids. Editors R. Manske and H. Holmes (New York: Academic Press), 24.

UMSP (2010). UMSP sentinel Site Malaria Surveillance Report. Uganda Malaria Surveillance Project.

Upadhyay, H. C., Sisodia, B. S., Agrawal, J., Pal, A., Darokar, M. P., and Srivastava, S. K. (2014). Antimalarial Potential of Extracts and Isolated Compounds from 
Four Species of Genus Ammannia. Med. Chem. Res. 23, 870-876. doi:10.1007/ s00044-013-0682-5

Uwimana, A., Legrand, E., Stokes, B. H., Ndikumana, J. M., Warsame, M., Umulisa, N., et al. (2020). Emergence and Clonal Expansion of In Vitro Artemisinin-Resistant Plasmodium Falciparum Kelch13 R561H Mutant Parasites in Rwanda. Nat. Med. 26, 1602-1608. doi:10.1038/s41591-020$1005-2$

Valadares, M. C., Rezende, K. R., Pereira, E. R., Sousa, M. C., Gonçalves, B., de Assis, J. C., et al. (2007). Protective Effects of 4-nerolidylcatechol against Genotoxicity Induced by Cyclophosphamide. Food Chem. Toxicol. 45, 1975-1978. doi:10.1016/j.fct.2007.04.016

Van Agtmael, M. A., Eggelte, T. A., and Van Boxtel, C. J. (1999). Artemisinin Drugs in the Treatment of Malaria: from Medicinal Herb to Registered Medication. Trends Pharmacol. Sci. 20, 199-205. doi:10.1016/S01656147(99)01302-4

Van Vuuren, S., and Frank, L. (2020). Review: Southern African Medicinal Plants Used as Blood Purifiers. J. Ethnopharmacol. 249, 112434. doi:10.1016/ j.jep.2019.112434

Van Wyk, B. E. (2015). A Review of Commercially Important African Medicinal Plants. J. Ethnopharmacol. 176, 118134. doi:10.1016/j.jep.2015.10.031

Wasunna, B., Zurovac, D., Goodman, C. A., and Snow, R. W. (2008). Why Don't Health Workers Prescribe ACT? A Qualitative Study of Factors Affecting the Prescription of Artemether-Lumefantrine. Malar. J. 7, 29. doi:10.1186/14752875-7-29

Weissbuch, I., and Leiserowitz, L. (2008). Interplay between Malaria, Crystalline Hemozoin Formation, and Antimalarial Drug Action and Design. Chem. Rev. 108, 4899-4914. doi:10.1021/cr078274t

WHO (2018b). Artemisinin Resistance and Artemisinin-Based Combination Therapy Efficacy: Status Report. Switzerland: World Health Organization. Available at: https://apps.who.int/iris/handle/10665/274362 (Accessed June 21, 2021)

WHO (2015). Guidelines for the Treatment of Malaria. 3rd Edn. Switzerland: World Health Organization. Available at: https://www.afro.who.int/ publications/guidelines-treatment-malaria-third-edition (Accessed June 21, 2021).

WHO (1998). Medicinal Plants in the Republic of Korea: Information on 150 Commonly Used Medicinal Plants. Philippines: World Health Organization Regional Publications, Regional Office for the Western Pacific, Manila. Available at: https://apps.who.int/iris/handle/10665/207585 (Accessed June 21, 2021)

WHO (2017). Status Report on Artemisinin and ACT Resistance Efficacy. Switzerland: World Health Organization. Available from: https://www. who.int/publications/i/item/WHO-CDS-GMP-2019.17 (Accessed June 21, 2021).

WHO (2020b). The World Malaria Report, the Malaria Atlas Project. Switzerland: World Health Organization. Available at: https://malariaatlas.org/trends/ region (Accessed June 29, 2021).

WHO (2018a). World Malaria Report 2018 Ethiopia. Switzerland: World Health Organization. Available from: https://www.who.int/malaria/publications/ country-profiles/profile_eth_en.pdf?ua=1 (Accessed June 21, 2021).

WHO (2019). World Malaria Report 2019. Switzerland: World Health Organization. Available from: https://www.who.int/publications/i/item/ 9789241565721 (Accessed June 21, 2021).

WHO (2020a). World Malaria Report 2020. Switzerland: World Health Organization. Available from: https://www.who.int/publications/i/item/ 9789240015791 (Accessed June 21, 2021).
Wicht, K. J., Mok, S., and Fidock, D. A. (2020). Molecular Mechanisms of Drug Resistance in Plasmodium Falciparum Malaria. Annu. Rev. Microbiol. 74, 431-454. doi:10.1146/annurev-micro-020518-115546

Willcox, M. (2011). Improved Traditional Phytomedcines in Current Use for the Clinical Treatment of Malaria. Planta Med. 77, 662-671. doi:10.1055/s-00301250548

Willcox, M., Bodeker, G., Bourdy, G., Dhingra, V., Falquet, J., Ferreira, J. F. S., et al. (2003). "Artemisia Annua as a Traditional Herbal Antimalarial,"in Traditional Medicinal Plants and Malaria. Editors M. Willcox, G. Bodeker, and P. Rasoanaivo (Boca Raton: CRC Press), 43-59.

Willcox, M., Burford, G., and Bodeker, G. (2004). "An Overview of Ethnobotanical Studies on Plants Used for the Treatment of Malaria," in Traditional Medicinal Plants and Malaria. Editors M. Willcox, G. Bodeker, and P. Rasoanaivo (Boca Raton: CRC Press), 187-197.

Willcox, M. L., Graz, B., Falquet, J., Diakite, C., Giani, S., and Diallo, D. (2011). A "Reverse Pharmacology" Approach for Developing an Anti-malarial Phytomedicine. Malar. J. 10, S8. doi:10.1186/1475-2875-10-S1-S8

Wondafrash, D. Z., Bhoumik, D., Altaye, B. M., Tareke, H. B., and Assefa, B. T. (2019). Antimalarial Activity of Cordia Africana (Lam.) (Boraginaceae) Leaf Extracts and Solvent Fractions in Plasmodium Berghei-infected Mice. Evidbased Compl. Alt. Med. 2019, 8324596. doi:10.1155/2019/8324596

Yeka, A., Kigozi, R., Conrad, M. D., Lugemwa, M., Okui, P., Katureebe, C., et al. (2016). Artesunate/amodiaquine versus Artemether/lumefantrine for the Treatment of Uncomplicated Malaria in Uganda: A Randomized Trial. J. Infect. Dis. 213, 1134-1142. doi:10.1093/infdis/jiv551

Yi, J., Ye, X., Wang, D., He, K., Yang, Y., Liu, X., et al. (2013). Safety Evaluation of Main Alkaloids from Rhizoma Coptidis. J. Ethnopharmacol. 145, 303-310. doi:10.1016/j.jep.2012.10.062

Youyou, T. (2015). Facts and Biography: The Nobel Prize in Physiology or Medicine 2015. Solna, Sweden: The Nobel Foundation. Available at: https://www. nobelprize.org/prizes/medicine/2015/tu/facts/ (Accessed May 3, 2021).

Zalis, M. G., Pang, L., Silveira, M. S., Milhous, W. K., and Wirth, D. F. (1998). Characterization of Plasmodium Falciparum Isolated from the Amazon Region of Brazil: Evidence for Quinine Resistance. Am. J. Trop. Med. Hyg. 58, 630-637. doi:10.4269/ajtmh.1998.58.6310.4269/ajtmh.1998.58.630

Zhou, L., Xu, J., and Peng, Y. (2014). "Medicinal Crops," in Encyclopedia of Agriculture and Food Systems. Editor N. K. V. Alfen (San Diego: Elsevier), 223-230. doi:10.1016/b978-0-444-52512-3.00152-2

Conflict of Interest: The authors declare that the research was conducted in the absence of any commercial or financial relationships that could be construed as a potential conflict of interest.

Publisher's Note: All claims expressed in this article are solely those of the authors and do not necessarily represent those of their affiliated organizations, or those of the publisher, the editors and the reviewers. Any product that may be evaluated in this article, or claim that may be made by its manufacturer, is not guaranteed or endorsed by the publisher.

Copyright $\odot 2021$ Ceravolo, Aguiar, Adebayo and Krettli. This is an open-access article distributed under the terms of the Creative Commons Attribution License (CC $B Y$ ). The use, distribution or reproduction in other forums is permitted, provided the original author(s) and the copyright owner(s) are credited and that the original publication in this journal is cited, in accordance with accepted academic practice. No use, distribution or reproduction is permitted which does not comply with these terms. 